\title{
Comparison of holographic and field theoretic complexities for time dependent thermofield double states
}

\author{
Run-Qiu Yang, ${ }^{a}$ Chao Niu, ${ }^{b}$ Cheng-Yong Zhang ${ }^{c, d}$ and Keun-Young $\mathrm{Kim}^{b}$ \\ ${ }^{a}$ Quantum Universe Center, Korea Institute for Advanced Study, \\ Seoul 130-722, South Korea \\ ${ }^{b}$ School of Physics and Chemistry, Gwangju Institute of Science and Technology, \\ Gwangju 61005, South Korea \\ ${ }^{c}$ Department of Physics and State Key Laboratory of Nuclear Physics and Technology, \\ Peking University, \\ 5 Yiheyuan Road, Beijing 100871, China \\ ${ }^{d}$ Center for High Energy Physics, Peking University, \\ 5 Yiheyuan Road, Beijing 100871, China \\ E-mail: aqiu@kias.re.kr, chaoniu09@gmail.com, zhangcy0710@pku.edu.cn, \\ fortoe@gist.ac.kr
}

ABSTRACT: We compute the time-dependent complexity of the thermofield double states by four different proposals: two holographic proposals based on the "complexity-action" (CA) conjecture and "complexity-volume" (CV) conjecture, and two quantum field theoretic proposals based on the Fubini-Study metric (FS) and Finsler geometry (FG). We find that four different proposals yield both similarities and differences, which will be useful to deepen our understanding on the complexity and sharpen its definition. In particular, at early time the complexity linearly increase in the CV and FG proposals, linearly decreases in the FS proposal, and does not change in the CA proposal. In the late time limit, the $\mathrm{CA}, \mathrm{CV}$ and FG proposals all show that the growth rate is $2 E /(\pi \hbar)$ saturating the Lloyd's bound, while the FS proposal shows the growth rate is zero. It seems that the holographic CV conjecture and the field theoretic FG method are more correlated.

KEYWORDS: Gauge-gravity correspondence, Holography and condensed matter physics (AdS/CMT)

ArXIV EPRINT: 1710.00600 


\section{Contents}

1 Introduction 1

2 Holographic complexity potential 3

3 Time dependent complexity of the TFD states: holographic approach 6

3.1 CA conjecture 6

3.1.1 Total action with the boundary and joint terms 6

$\begin{array}{lll}3.1 .2 & \text { Complexity potential } & 8\end{array}$

$\begin{array}{ll}3.1 .3 & \text { Time dependnent complexity } \\ \end{array}$

$\begin{array}{lll}3.2 & \text { CV conjecture } & 14\end{array}$

4 Time dependent complexity of the TFD states: field theory approach 18

$\begin{array}{ll}4.1 \text { Time evolution of the TFD states } & 18\end{array}$

$\begin{array}{lll}4.2 & \text { Fubini-Study (FS) metric } & 21\end{array}$

4.3 Finsler geometry (FG) 24

$\begin{array}{llr}5 & \text { Summary } & 28\end{array}$

$\begin{array}{ll}\text { A Bogoliubov transformation and TFD states } & 31\end{array}$

B Fubini-Study metric $\quad 32$

$\begin{array}{lll}\text { C Finsler structure in the generator set } E_{L} & 32\end{array}$

D Complexity of operator generated by $E_{L} \quad 34$

\section{Introduction}

The applications of the holographic principle on the study of black holes have lead to many new surprising discoveries. In particular, it has been shown that the quantum information may play important roles in the quantum gravity. One important discovery is the connection between entanglement and geometry [1-4]. It inspired a viewpoint that a black hole might be highly entangled with a system that was effectively infinitely far away. This viewpoint lead Juan Maldacena and Leonard Susskind to propose a very interesting conjecture named "ER=EPR" [3] when they considered the wormhole created by an Einstein-Rosen (ER) bridge [5] and a pair of maximally entangled black holes. Here EPR refers to quantum entanglement (Einstein-Podolsky-Rosen paradox). To understand how much difficult sending a signal through the ERB from one side to the other, a new information-theoretic quantity named "complexity" was imported into the holographic duality and quantum 
gravity $[6,7]$. Basically, the complexity describes how many fundamental gates or operators are required when we try to obtain a target state from a reference state.

In order to construct holographic models to describe the complexity, let us consider the following thermofield double (TFD) state

$$
|\mathrm{TFD}\rangle:=Z^{-1 / 2} \sum_{\alpha} \exp \left[-E_{\alpha} /(2 T)\right]\left|E_{\alpha}\right\rangle_{L}\left|E_{\alpha}\right\rangle_{R}
$$

The states $\left|E_{\alpha}\right\rangle_{L}$ and $\left|E_{\alpha}\right\rangle_{R}$ are defined in the two copy CFTs and $T$ is the temperature of these two CFTs. This state is conjectured to be approximately dual to an eternal AdS black hole with the Hawking temperature which is the same as $T$ of CFTs [8]. With the Hamiltonians $H_{L}$ and $H_{R}$ at the left and right dual CFTs, respectively, the time evolution of a TFD state

$$
\left|\psi\left(t_{L}, t_{R}\right)\right\rangle:=e^{-i\left(t_{L} H_{L}+t_{R} H_{R}\right)}|\mathrm{TFD}\rangle,
$$

can be characterized by the codimension-two surface of $t=t_{L}$ and $t=t_{R}$ at the two boundaries of the AdS black hole [8,9]. $H_{L}$ and $H_{R}$ are short for $H_{L} \otimes \mathbf{1}_{R}$ and $\mathbf{1}_{L} \otimes H_{R}$ respectively. Even though we introduced two times $t_{L}$ and $t_{R}$, our system will depend only on the combination $\bar{t}:=t_{L}+t_{R}$ and $\bar{t}$ plays a role of field theory time. For a symmetric configuration we may identify $t_{L}=t_{R}=t / 2$ so that $\bar{t}=t$. Equivalently, we may consider a total Hamiltonian $H_{\text {total }}=\mathbf{1}_{L} \otimes H_{R}$ with a field theory time $t_{R}$.

Two different conjectures were proposed to compute the complexity of $\left|\psi\left(t_{L}, t_{R}\right)\right\rangle$ holographically: ${ }^{1}$ the $\mathrm{CV}($ complexity= volume) conjecture $[6,10,13]$ and the $\mathrm{CA}($ complexity $=$ action) conjecture $[9,14]$.

The CV conjecture states that the complexity of $\left|\psi\left(t_{L}, t_{R}\right)\right\rangle$ is proportional to the maximal volume of the space-like codimension-one surface which connects the codimensiontwo time slices denoted by $t_{L}$ and $t_{R}$ at two AdS boundaries, i.e.

$$
\mathcal{C}_{V}=\max _{\partial \Sigma=t_{L} \cup t_{R}}\left[\frac{V(\Sigma)}{G_{N} \ell}\right]
$$

where $G_{N}$ is the Newton's constant. $\Sigma$ is a possible space-like codimension-one surface which connects $t_{L}$ and $t_{R}$. $\ell$ is a length scale associated with the bulk geometry such as the horizon radius or AdS radius and so on. However, there is an ambiguity coming from the choice of a length scale $\ell$. This unsatisfactory feature motivated the second conjecture: the CA conjecture [9, 14], which says the complexity of $\left|\psi\left(t_{L}, t_{R}\right)\right\rangle$ is dual to the action in the Wheeler-DeWitt (WDW) patch associated with $t_{L}$ and $t_{R}$, i.e.

$$
\mathcal{C}_{A}=\frac{I_{\mathrm{WDW}}}{\pi \hbar} .
$$

The WDW patch associated with $t_{L}$ and $t_{R}$ is the collection of all space-like surface connecting $t_{L}$ and $t_{R}$ with the null sheets coming from $t_{L}$ and $t_{R}$. More precisely it is the domain of dependence of any space-like surface connecting $t_{L}$ and $t_{R}$.

\footnotetext{
${ }^{1}$ There are also other holographic proposals for complexity, see refs. [10-12] for examples.
} 
Recently, two different methods were proposed by refs. $[15,16]$ to define the complexity in quantum field theory. ${ }^{2}$ The method in ref. [15], which we will call the FS method in this paper, is based on the Fubini-Study metric and defined the complexity of two states to be the length of the geodesic under the Fubini-Study metric. The method in ref. [16], which we will call the FG method in this paper, first defined the complexity for operators by the Finsler geometry ${ }^{3}$ and then used this complexity to define the complexity between states. As what we will present later, these two methods are very suitable to compute the complexity between two different TFD states.

Taking the eqs. (1.3), (1.4) and two quantum field theory proposals in refs. [15, 16] into account, we have at least four different methods to compute the complexity between two TFD states. The goal of this paper is to compute the complexity in four different methods and see their similarities and differences. It may give us some information to judge which are appropriate methods to compute the complexity among four methods. It may also shed light on a possible connection between two holographic conjectures and two quantum field theory proposals.

The paper is organized as follows. In section 2 we introduce several concepts for complexity in literature and clarify some subtle issues in defining the complexity regarding the reference state and divergence in holographic complexity. In section 3 we use the modified $\mathrm{CA}$ and $\mathrm{CV}$ conjectures introduce in section 2 to compute complexity between the time dependent TFD state and their corresponding zero temperature vacuum state. In section 4, after presenting how to construct a time-dependent TFD state for free field theory explicitly, we use two different quantum field theoretic proposals to compute the complexity between the time-dependent TFD state and the vacuum. Our computations by four different methods are summarized and compared in section 5 .

Note added. While this work was being finished, ref. [23] appeared which also studied the complexity growth rate. Our results in section 3 have some overlap with ref. [23].

\section{Holographic complexity potential}

Before we compute the holographic time-dependent complexity, let us first make some comments on the CV and CA conjectures and present our modified versions of them.

Although these two conjectures satisfy important requirements on the complexity such as the Lloyd's bound [24-27], it seems that there are a few subtle issues to be clarified. First, the complexity computed by eq. (1.3) or eq. (1.4) is infinite. ${ }^{4}$ Second, a possibly related issue is an ambiguity for the reference state. The complexity should be defined between any two states, say the target state and the reference state. However, for both original holographic complexities ( $\mathrm{CV}$ and $\mathrm{CA}$ ) it is not clear what the reference state is. An

\footnotetext{
${ }^{2}$ Refs. $[17,18]$ also give a definition for the complexity in conformal field theory by the Liouville action. It is also interesting to see what the results are if this method is applied to the time-dependent TFD states.

${ }^{3}$ The Finsler geometry was first introduced to investigate the computational complexity by refs. [19-21] and recently drew attention again in ref. [22].

${ }^{4}$ The divergence of the complexity computed by the CV and CA conjectures show some intrinsic properties of CFT similarly to the case of the entanglement entropy [28].
} 
unknown privileged reference state seems assumed. By proposing "modified complexity" we want to define the complexity between any two arbitrary states without a privileged reference state.

Even if we accept this unknown privileged reference state ${ }^{5}$ exists, it seems that the original CA conjecture has two more issues. First, in principle, the complexity between two states should be non-negative but the value computed by eq. (1.4) can be negative. Second, the dynamics will be invariant if we add a constant term into the action so the complexity should also be invariant after adding a constant term into the gravity action. However, the original CA conjecture does not satisfy this property.

To resolve this issue a "modified" complexity was proposed in ref. [16]. It suggests that the original CV and CA conjectures (1.3) and (1.4) give a kind of "complexity potential" rather than the complexity between any two states. When we restrict our considerations to the TFD states, the leading order of complexity between two TFD states $\left|\mathrm{TFD}_{1}\right\rangle$ and $\left|\mathrm{TFD}_{2}\right\rangle$ is given by the following formulas in the $\mathrm{CV}$ or $\mathrm{CA}$ conjectures ${ }^{6}$

$$
\begin{aligned}
& \mathcal{C}_{V}\left(\left|\mathrm{TFD}_{2}\right\rangle,\left|\mathrm{TFD}_{1}\right\rangle\right)=\left|\mathcal{C}_{V}^{(1)}-\mathcal{C}_{V}^{(2)}\right|, \\
& \mathcal{C}_{A}\left(\left|\mathrm{TFD}_{2}\right\rangle,\left|\mathrm{TFD}_{1}\right\rangle\right)=\left|\mathcal{C}_{A}^{(1)}-\mathcal{C}_{A}^{(2)}\right| .
\end{aligned}
$$

Here $\left\{\mathcal{C}_{V}^{(1)}, \mathcal{C}_{V}^{(2)}\right\}$ and $\left\{\mathcal{C}_{A}^{(1)}, \mathcal{C}_{A}^{(2)}\right\}$ are computed by eqs. (1.3) or (1.4). Two TFD states have different properties such as temperatures, charges or other thermodynamic quantities. In holographic perspective, a TFD state is determined by the two time slices $\left(t_{R}, t_{L}\right)$ at the two boundaries in a black hole.

This modification does not lose any important physical properties of the original version and seems simpler because it does not need to refer to an unknown reference state. ${ }^{7}$ This modified version has the following basic properties:

- Triangle inequality: $\mathcal{C}\left(\left|\mathrm{TFD}_{2}\right\rangle,\left|\mathrm{TFD}_{0}\right\rangle\right)+\mathcal{C}\left(\left|\mathrm{TFD}_{0}\right\rangle,\left|\mathrm{TFD}_{1}\right\rangle\right) \geq \mathcal{C}\left(\left|\mathrm{TFD}_{2}\right\rangle,\left|\mathrm{TFD}_{1}\right\rangle\right.$ for any state $\left|\mathrm{TFD}_{0}\right\rangle$

- Reversibility: $\mathcal{C}\left(\left|\mathrm{TFD}_{2}\right\rangle,\left|\mathrm{TFD}_{1}\right\rangle\right)=\mathcal{C}\left(\left|\mathrm{TFD}_{1}\right\rangle,\left|\mathrm{TFD}_{2}\right\rangle\right)$

In particular, when eq. (2.1) is applied to the TFD states, the results in eq. (2.1) agree to the results obtained in a quantum field theory approach in ref. [16] and also agree

\footnotetext{
${ }^{5}$ Because the complexity from any state to itself is zero, if this reference state is any state dual to an asymptotic AdS black hole then we can find eq. (1.3) or (1.4) should be zero at this black hole. However, ref. [28] has proven that eqs. (1.3) or (1.4) are divergent for all asymptotically AdS black holes, so this reference state is not dual to any AdS black hole.

${ }^{6}$ Formally, eq. (2.1) is similar to the "complexity of formation" proposed by ref. [29]. However, they are not always the same. A detailed discussion about it can be found in ref. [16].

${ }^{7}$ In principle, a well-defined complexity should satisfy that $\mathcal{C}\left(\left|\mathrm{TFD}_{2}\right\rangle,\left|\mathrm{TFD}_{1}\right\rangle\right)=0$ if and only if $\left|\mathrm{TFD}_{2}\right\rangle=\left|\mathrm{TFD}_{1}\right\rangle$. However, it may be possible that the modified version in eq. (2.1) vanish even with two different states. This can appear if the system has multiple different solutions for given physical conditions, for example, in the cases which contain phase transitions. Another possibility stems from the the fact that the TFD states is only approximately dual to the eternal AdS black holes so we can expect the original CA and CV conjectures in eq. (1.3) and (1.4) may lose some subleading contributions.
} 
to the results computed by the method proposed in ref. [15] (which will be presented in subsection 4.2).

The formula (2.1) can be understood more geometrically. Let us assume a space where all states can be parameterized by $x^{a}$, which may be the temperature, total charge, mass or any other quantity describing different time slices at two boundaries. Suppose that there is a curve $l: x^{a}=x^{a}(\lambda)$ which satisfies $\left|\operatorname{TFD}\left(x^{a}\left(\lambda_{1}\right)\right)\right\rangle=\left|\operatorname{TFD}_{1}\right\rangle$ and $\left|\operatorname{TFD}\left(x^{a}\left(\lambda_{2}\right)\right)\right\rangle=\left|\operatorname{TFD}_{2}\right\rangle$ with $\lambda_{1} \leq \lambda_{2}$. For any given curve $l$, we can use eqs. (1.3) or (1.4) to compute the $\mathcal{C}_{V}(\lambda)$ and $\mathcal{C}_{A}(\lambda)$, which are the functions of $\lambda$ and depend on the choice of the curve $l$. Then the complexity between $\left|\mathrm{TFD}_{1}\right\rangle$ and $\left|\mathrm{TFD}_{2}\right\rangle$ is given by ${ }^{8}$

$$
\mathcal{C}_{V}\left(\left|\mathrm{TFD}_{2}\right\rangle,\left|\mathrm{TFD}_{1}\right\rangle\right)=\min \left\{\int_{\lambda_{1}}^{\lambda_{2}}\left|\frac{\mathrm{d} \mathcal{C}_{V}(\lambda)}{\mathrm{d} \lambda}\right| \mathrm{d} \lambda \mid \forall l: x^{a}=x^{a}(\lambda)\right\},
$$

and

$$
\mathcal{C}_{A}\left(\left|\mathrm{TFD}_{2}\right\rangle,\left|\mathrm{TFD}_{1}\right\rangle\right)=\min \left\{\int_{\lambda_{1}}^{\lambda_{2}}\left|\frac{\mathrm{d} \mathcal{C}_{A}(\lambda)}{\mathrm{d} \lambda}\right| \mathrm{d} \lambda \mid \forall l: x^{a}=x^{a}(\lambda)\right\} .
$$

These two equations in fact give the Finsler structures such that for any tangent vector $T^{a}=(\partial / \partial \lambda)^{a}=\mathrm{d} x^{a} / \mathrm{d} \lambda$

$$
F\left(x^{a}, T^{a}\right):=\left|\left(\mathbf{d} \mathcal{C}_{X}\right)_{a} T^{a}\right|,
$$

where $X=V$ or $A$ and $\mathbf{d}$ is the exterior differential operator in the parameter space spanned by $x^{a}$. The integrations in eqs. (2.2) and (2.3) give a holographic version of "complexity geometry", which is similar to the FG and FS methods in refs. [15, 16, 29]. Since the absolute value appearing in these two formulas is not convenient, we introduce a positive infinitesimal value $\epsilon$ and arbitrary functions $\omega_{a b}$ so that eqs. (2.2) and (2.3) can be written as the following form

$$
\mathcal{C}_{X}\left(\left|\mathrm{TFD}_{2}\right\rangle,\left|\mathrm{TFD}_{1}\right\rangle\right)=\lim _{\epsilon \rightarrow 0^{+}} \min \left\{\int_{\lambda_{1}}^{\lambda_{2}} \sqrt{\left(\frac{\partial \mathcal{C}_{X}}{\partial x^{a}} \frac{\mathrm{d} x^{a}}{\mathrm{~d} \lambda}\right)^{2}+\epsilon^{2} \omega_{a b} \frac{\mathrm{d} x^{a}}{\mathrm{~d} \lambda} \frac{\mathrm{d} x^{b}}{\mathrm{~d} \lambda}} \mathrm{d} \lambda\right\} .
$$

It is assumed that the limit is well defined and independent of the choices of auxiliary functions $\omega_{a b}$. This means that we have the following "holographic complexity metric" defined in a parameter space spanned by $x^{a}$,

$$
\mathrm{d} s_{X}^{2}:=\left[\frac{\partial \mathcal{C}_{X}}{\partial x^{a}} \frac{\partial \mathcal{C}_{X}}{\partial x^{b}}+\epsilon^{2} \omega_{a b}\right] \mathrm{d} x^{a} \mathrm{~d} x^{b},
$$

Since the metric defined in eq. (2.6) is a tensor the complexity is diffeomorphism invariant under the reparameterizations on $x^{a}$. The minimal values of eq. (2.2) then is the lengths of geodesics given by metric $(2.6)$ if $\mathcal{C}_{V}\left(x^{a}\right)$ and $\mathcal{C}_{A}\left(x^{a}\right)$ are $C^{2}$ functions of $x^{a}$.

To show that eqs. (2.2) and (2.3) are equivalent to eq. (2.1) note that

$$
\int_{\lambda_{1}}^{\lambda_{2}} \mathrm{~d} \lambda\left|\frac{\mathrm{d} \mathcal{C}_{X}}{\mathrm{~d} \lambda}\right|=\int_{l}\left|\mathrm{~d} \mathcal{C}_{X}\right| \geq\left|\int_{l} \mathrm{~d} \mathcal{C}_{X}\right|=\left|\mathcal{C}_{X}^{(1)}-\mathcal{C}_{X}^{(2)}\right|
$$

\footnotetext{
${ }^{8}$ We assume that the curve is regular, which means that $0 \leq\left|\partial x^{a} / \partial \lambda\right|<\infty$.
} 
The equality can be achieved if there is a curve with its tangent $T^{a}$ satisfying,

$$
\left(\mathbf{d} \mathcal{C}_{X}\right)_{a} T^{a} \leq 0, \quad \forall \lambda \in\left(\lambda_{1}, \lambda_{2}\right), \quad \text { or } \quad\left(\mathbf{d} \mathcal{C}_{X}\right)_{a} T^{a} \geq 0, \quad \forall \lambda \in\left(\lambda_{1}, \lambda_{2}\right) .
$$

For the case that parameter space has trivial topology and its dimensional is larger than 1 , such a curve always can be found, so eq. (2.7) shows,

$$
\mathcal{C}_{X}\left(\left|\mathrm{TFD}_{1}\right\rangle,\left|\mathrm{TFD}_{2}\right\rangle\right)=\left|\mathcal{C}_{X}^{(1)}-\mathcal{C}_{X}^{(2)}\right|
$$

which is eq. (2.1). However, for the cases that parameter space is one dimensional or has non-trivial topology, the condition (2.8) may not be achieved. The complexity modified definitions (2.2) and (2.3) may different from ones in eq. (2.9). In these cases, one have to use definitions (2.2) and (2.3) to compute the complexity in holography.

Let us make a summary regarding several concepts for the complexity introduced in literature and this paper. If we can compute the "complexity potential" $\mathcal{C}_{V}\left(x^{a}\right)$ and $\mathcal{C}_{A}\left(x^{a}\right)$ by eqs. (1.3) and (1.4), which is the original proposals, then we can obtain the "modified complexity" which is the complexity between two states through eq. (2.9). The holographic complexity potential has an additional freedom: if we add any term independent of $x^{a}$, the modified complexity will be invariant. This freedom gives us a possibility to introduce suitable subtraction terms to renormalize the divergent holographic complexity potential defined by eqs. (1.3) and (1.4). This is the foundation of the "regularized holographic complexity" proposed by ref. [30], which we will call "renormalized complexity potential" in this paper.

\section{Time dependent complexity of the TFD states: holographic approach}

In this section, we will use the modified CV and CA conjectures to compute the complexity between the time-dependent TFD states and their corresponding vacuum states. There are three parameters in the holographic duals: the temperature of the bulk black hole, the time of the left boundary and the time of the right boundary slice i.e. $\left\{T, t_{L}, t_{R}\right\}$. We first have to compute the complexity potential $\mathcal{C}_{V}\left(T, t_{L}, t_{R}\right)$ and $\mathcal{C}_{A}\left(T, t_{L}, t_{R}\right)$ which are divergent. To deal with this divergence, ref. [30] proposed a method to renormalize them by adding some counterterms. In the planner symmetry AdS black holes, the general counterterms have been found and are independent of the values of $T, t_{L}$ and $t_{R}$. Therefore, we can use the renormalized complexity potential for the holographic complexity potential and finally find the modified complexity between two TFD states. In the following subsections, we will perform these procedures in both the $\mathrm{CA}$ and $\mathrm{CV}$ conjectures.

Since we focus on the TFD states which are dual to the planar symmetric Schwarzschild AdS black holes, the problems can be simplified. Thanks to the time translation symmetry, the systems only depend on $t_{L}+t_{R}$ so we are left with only two independent parameters $\left\{T, t_{L}+t_{R}\right\}$.

\subsection{CA conjecture}

\subsubsection{Total action with the boundary and joint terms}

The central issue in the CA conjecture is the computation of the on-shell action. Because the null boundary and joint terms are involved in this computation, it was a subtle problem 
at the time when this conjecture was proposed. However, after several careful analysis on the action with null boundaries [31-34], the action in general relativity with suitable boundary and joint terms turned out to be the following form,

$$
I=\frac{1}{16 \pi} \int_{\mathcal{M}} \mathrm{d}^{d+1} x \sqrt{-g}\left[R+\frac{d(d-1)}{\ell_{\text {AdS }}^{2}}\right]+\sum_{i} I_{B_{i}}+\sum_{j} I_{\mathcal{N}_{j}}+\sum I_{\mathcal{J}}+\sum I_{\mathcal{J}^{\prime}} .
$$

Here $R$ is the scalar curvature in the bulk, $\ell_{\text {AdS }}$ is the AdS radius, $I_{B_{i}}$ is the GibbonsHawking-York (GHY) boundary term for the non-null boundary fragments $B_{i}, I_{\mathcal{N}_{j}}$ is the boundary term for the null boundary fragments $\mathcal{N}_{j}, I_{\mathcal{J}}$ is the joint term defined on the joint of two non-null boundaries and $I_{\mathcal{J}^{\prime}}$ is the joint term defined on the joint intersected by the null boundaries and others.

The first result for the null boundary term was proposed by ref. [31] and then represented by refs. [32-34]. Refs. [31, 34] showed that the suitable null boundary term should be

$$
I_{\mathcal{N}_{j}}=I_{\mathcal{N}_{j}}^{(1)}:=-\frac{\operatorname{sign}\left(\mathcal{N}_{j}\right)}{8 \pi} \int_{\mathcal{N}_{j}} \mathrm{~d} \lambda \mathrm{d}^{d-2} x \sqrt{|\sigma|}(\kappa+\Theta),
$$

where $\lambda$ is the integral curve of the normal vector $k^{\mu}$ (future directed) for the null boundary, i.e., $k^{\mu}=(\partial / \partial \lambda)^{\mu}$. $\kappa$ is the "surface gravity" of the null surface corresponding to $k^{\mu}$ and satisfies $k^{\mu} \nabla_{\mu} k^{\nu}=\kappa k^{\nu} . \operatorname{sign}\left(\mathcal{N}_{j}\right)$ is +1 only when it lies on the future boundary of $\mathcal{M}$, otherwise, $\operatorname{sign}\left(\mathcal{N}_{j}\right)$ is $-1 . \sigma$ is the determinant of the induced metric at the transverse codimensional 2 surface orthogonal to $k^{\mu}$. $\Theta$ is the expansion of the null boundary measured by $\lambda$ and satisfies $\Theta=(\sqrt{|\sigma|})^{-1} k^{\mu} \partial_{\mu} \sqrt{|\sigma|}$. Considering the fact that $\Theta$ itself vanishes during the variation, refs. $[32,33]$ proposed the "minimal null boundary term" by dropping the expansion term in eq. (3.2), which reads

$$
I_{\mathcal{N}_{j}}=I_{\mathcal{N}_{j}}^{(2)}:=-\frac{\operatorname{sign}\left(\mathcal{N}_{j}\right)}{8 \pi} \int_{\mathcal{N}_{j}} \mathrm{~d} \lambda \mathrm{d}^{d-2} x \sqrt{|\sigma|} \kappa
$$

However, the boundary terms (3.2) and (3.3) are both dependent on the choice of $\lambda$ so the re-parameterization of $\lambda$ can lead different values for the null boundary term. To overcome this problem, refs. $[32,35]$ proposed that we should add an additional term into the boundary term $(3.3)$

$$
I_{\mathcal{N}_{j}}=I_{\mathcal{N}_{j}}^{(3)}:=-\frac{\operatorname{sign}\left(\mathcal{N}_{j}\right)}{8 \pi} \int_{\mathcal{N}_{j}} \mathrm{~d} \lambda \mathrm{d}^{d-2} x \sqrt{|\sigma|}\left(\kappa+L_{0}\right),
$$

where $L_{0}=\Theta \ln \left(|\Theta| / \ell_{\text {AdS }}\right)$ if $\mathcal{N}$ lies to the future boundary of $\mathcal{M}$. Otherwise, $L_{0}=$ $-\Theta \ln \left(|\Theta| / \ell_{\text {AdS }}\right)$. In this paper, we will use this total boundary term.

The joint term $I_{\mathcal{J}}$ was first found by ref. [36] and then confirmed by refs. [32, 34] again recently by different methods. As the CA conjecture will not have such kind of joints, we will not give the detailed form for this kind of joint terms. The joint term for the case that there is at least one null boundary was first found by ref. [32], which is in general expressed as

$$
I_{\mathcal{J}^{\prime}}=\frac{\operatorname{sign}\left(\mathcal{J}^{\prime}\right)}{8 \pi} \int_{\mathcal{J}^{\prime}} \mathrm{d}^{d-1} x \sqrt{\sigma} a
$$


Here $\sigma$ is the determinant of the induced metric on the joint $\mathcal{J}^{\prime}$. According to the properties of the intersectional surface, the term $a$ can be computed as

$$
a=\left\{\begin{array}{l}
\ln \left(\left|n^{I} k_{I}\right|\right), \\
\ln \left(\left|k^{I} \bar{k}_{I}\right| / 2\right),
\end{array}\right.
$$

where $n^{I}$ is the unit normal vector (outward/future directed) for the non-null intersecting boundary, and $\bar{k}^{I}$ is the other null normal vector (future directed) for the null intersecting boundary. The value of $\operatorname{sign}\left(\mathcal{J}^{\prime}\right)= \pm 1$, which can be assigned as follows: " +1 " appears only when the WDW patch appears in the future/past of the null boundary component and the joint is at the past/future end of the null boundary fragments.

\subsubsection{Complexity potential}

The metric for the general $\operatorname{AdS}_{d+1}(d \geq 2)$ black hole is

$$
\mathrm{d} s^{2}=-r^{2} f(r) \mathrm{d} t^{2}+\frac{\mathrm{d} r^{2}}{r^{2} f(r)}+r^{2} \mathrm{~d} \Sigma_{d-1}^{2}
$$

where $\mathrm{d} \Sigma_{d-1}^{2}=\ell_{\mathrm{AdS}}^{-2} \sum_{i=1}^{d-1} \mathrm{~d} x_{i}^{2}$ is the $(d-1)$ dimensional line element and $\Sigma_{d-1}$ is the volume of the conformal boundary of the AdS black hole. The function $f(r)$ reads

$$
f(r)=\frac{1}{\ell_{\mathrm{AdS}}^{2}}\left(1-\frac{r_{h}^{d}}{r^{d}}\right) .
$$

The physical total mass (ADM mass) and temperature of this system are

$$
M=\frac{r_{h}^{d}(d-1) \Sigma_{d-1}}{16 \pi \ell_{\mathrm{AdS}}^{2}}, \quad T=\frac{r_{h} d}{4 \pi \ell_{\mathrm{AdS}}^{2}} .
$$

The Penrose diagram and the WDW patch are shown in figure 1. The time direction of the right boundary is the same as the coordinate time $t$ but the time of the left boundary is opposite to the coordinate time $t$. As the space-time has time translation symmetry the on-shell action only depends on the value of $t_{L}+t_{R}$. By this property we can fix $t_{L}=0$ and only study how the complexity depends on the value of $t_{R}$. In addition, thanks to the time reversal symmetry of the black hole we only consdier the case of $t_{R}>0$.

In figure 1 we see that there is a critical time $\Delta t_{c}$ distinguishing the left and right panel. Depending on the relationship between $t_{R}$ and $\Delta t_{c}$, there are two different types for the WDW patches. One is the case that $\left|t_{R}\right|<\Delta t_{c}$ shown in the left panel of figure 1 , where the two future and past null sheets all meet the singularities. The other one is the case that $\left|t_{R}\right|>\Delta t_{c}$ shown in the right panel of figure 1 , where the future directed null sheets coming from the boundaries will first meet the singularity $r=0$ but the past directed null sheets coming from the boundaries will meet each other in the inner region of black hole.

Let us introduce the infalling coordinate $v$ and outgoing coordinate $u$ as

$$
v=t+r^{*}, \quad u=t-r^{*},
$$



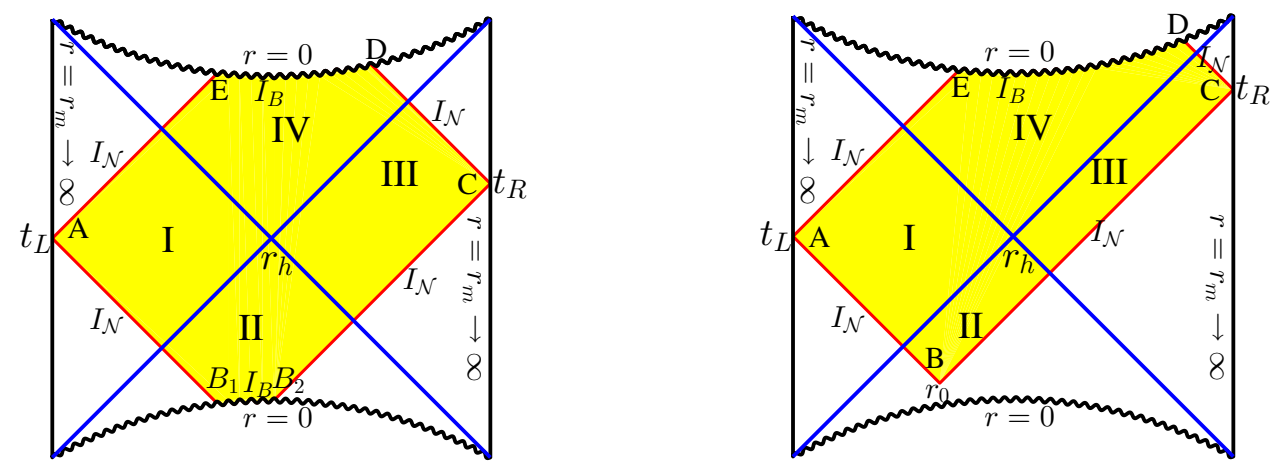

Figure 1. Penrose diagrams and WDW patches for $\operatorname{AdS}_{d+1}$ black hole $(d \geq 3)$ when $\left|t_{R}\right|<\Delta t_{c}$ (left panel) and $\left|t_{R}\right|>\Delta t_{c}$ (right panel). In the left panel, the past null sheets will meet the singularity at $B_{1}$ and $B_{2}$ respectively. In the right panel, the past null sheets will meet each other at $B$ with $r=r_{0} \in\left(0, r_{h}\right)$. In both panels, $I_{\mathcal{N}}$ and $I_{B}$ denote the null boundary terms and the spacelike boundary terms in (3.1) respectively. The joints $A, B_{1}, B_{2}, B, C, D$ and $E$ are the joints which involve at least one null boundary, so $I_{\mathcal{J}^{\prime}}$ in $(3.1)$ are associated with those joints. There is no contribution of $I_{\mathcal{J}}$ in (3.1).

where

$$
r^{*}(r)=\int^{r} \frac{\mathrm{d} x}{x^{2} f(x)}
$$

The null dual normal vector field for the null boundaries $A B$ and $C D$ is $k_{\mu}=-\left[(\mathrm{d} t)_{\mu}+\right.$ $\left.r^{-2} f^{-1}(\mathrm{~d} r)_{\mu}\right]$ and the null normal vector field for the null boundaries $B C$ and $A E$ is $\bar{k}_{\mu}=-\left[(\mathrm{d} t)_{\mu}-r^{-2} f^{-1}(\mathrm{~d} r)_{\mu}\right]$. These two null vector are affinely parameterized. The integration in eq. (3.11) can be expressed in terms of the hypergeometrical function

$$
r^{*}(r)=\frac{\ell_{\mathrm{AdS}}^{2}}{r}\left[{ }_{2} F_{1}\left(1,-\frac{1}{d} ; 1-\frac{1}{d} ; \frac{r^{d}}{r_{h}^{d}}\right)-1\right], \quad r<r_{h}
$$

and

$$
r^{*}(r)=\frac{\ell_{\mathrm{AdS}}^{2}}{r_{h}}\left[\cot \frac{\pi}{d}-\frac{r_{h}}{\left(r^{d}-r_{h}^{d}\right)^{1 / d}} 2 F_{1}\left(\frac{1}{d}, \frac{1}{d} ; 1+\frac{1}{d} ; \frac{r_{h}^{d}}{r_{h}^{d}-r^{d}}\right)-1\right], \quad r>r_{h} .
$$

The value of $\Delta t_{c}$ is given by,

$$
\Delta t_{c}:=2\left[r^{*}(\infty)-r^{*}(0)\right]=\frac{2 \ell_{\mathrm{AdS}}^{2}}{r_{h}} \frac{\pi}{d} \cot \frac{\pi}{d}
$$

To regularize the WDW patch, we assume the AdS boundaries are located at $r=r_{m} \gg$ $\ell_{\text {AdS }}$. One can see from figure 1 that when $t_{R} \leq \Delta t_{c}$, the WDW patch is the same as the one of $t_{R}=0$. This means that the corresponding TFD state is the same one for 
$t_{R} \in\left(0, \Delta t_{c}\right)$. So we have ${ }^{9}$

$$
\left|\operatorname{TFD}\left(T, t_{R}\right)\right\rangle=|\operatorname{TFD}(T, 0)\rangle, \text { if }\left|t_{R}\right| \leq \Delta t_{c},
$$

and

$$
\mathcal{C}_{\mathrm{A}, \mathrm{ren}}\left(t_{R}\right)=\mathcal{C}_{\mathrm{A}, \mathrm{ren}, 0},
$$

where $\mathcal{C}_{\mathrm{A} \text {,ren }}\left(t_{R}\right)$ is the renormalized complexity potential for given $t_{R}$ and $\mathcal{C}_{\mathrm{A} \text {,ren,0 }}$ is its value when $t_{R}=0$. The value of $\mathcal{C}_{\mathrm{A}, \text { ren }, 0}$ has been given by refs. [29, 30],

$$
\mathcal{C}_{\mathrm{A}, \mathrm{ren}, 0}=\frac{d-2}{d-1} \cot \left(\frac{\pi}{d}\right) \frac{M}{\hbar T} .
$$

The TFD state begins to evolve after $\left|t_{R}+t_{L}\right|>\Delta t_{c}$. In this case, the two past null sheets will meet each other at the joint $B$ with $r=r_{0} \in\left(0, r_{h}\right)$. We can obtain the equations for all the null boundaries, which are

$$
\begin{array}{rrr}
A B: & r_{m}^{*}=t+r^{*}(r), \\
C D: & t_{R}+r_{m}^{*}=t+r^{*}(r), \\
B C: & t_{R}-r_{m}^{*}=t-r^{*}(r), \\
A E: & -r_{m}^{*}=t-r^{*}(r) .
\end{array}
$$

By the equations for $A B$ and $B C$, we find that the past null sheets will meet each other at $r=r_{0}$, where $r_{0}$ is defined by the following equation

$$
\begin{aligned}
r^{*}\left(r_{0}\right) & =r_{m}^{*}-\frac{t_{R}}{2}, \quad r_{0}<r_{h} . \\
\frac{\ell_{\text {AdS }}^{2}}{r_{0}}\left[{ }_{2} F_{1}\left(1,-\frac{1}{d} ; 1-\frac{1}{d} ; \frac{r_{0}^{d}}{r_{h}^{d}}\right)-1\right] & =\frac{\ell_{\text {AdS }}^{2}}{r_{h}} \frac{\pi}{d} \cot \frac{\pi}{d}-\frac{t_{R}}{2} .
\end{aligned}
$$

For the case $d \geq 2$, this equation can be solved only numerically.

Now let us first compute the bulk contribution from the Einstein-Hilbert action. By using the Einstein's equation, we can write this term as

$$
I_{\text {bulk }}=\frac{1}{16 \pi} \int \mathrm{d}^{d+1} x \sqrt{-g}\left[R+\frac{d(d-1)}{\ell_{\mathrm{AdS}}^{2}}\right]=-\frac{\Sigma_{d-1} d}{8 \pi \ell_{\mathrm{AdS}}^{2}} \iint r^{d-1} \mathrm{~d} r \mathrm{~d} t .
$$

According to the right panel of figure 1, the bulk term can be splited into four parts. In the region I, for fixed $r$, the upper and inferior limits for $t$ in the integration (3.21) are given by the line equations $A B_{1}$ and $A E$, respectively. Thus we find

$$
I_{\text {bulk,I }}=-\frac{\Sigma_{d-1} d}{8 \pi \ell_{\text {AdS }}^{2}} \int_{r_{h}}^{r_{m}} r^{d-1} \mathrm{~d} r \int_{r^{*}(r)-r_{m}^{*}}^{r_{m}^{*}-r^{*}(r)} \mathrm{d} t .
$$

\footnotetext{
${ }^{9}$ Our result can be interpreted as the complexity of formation if we use the original CA conjecture with an unknown privileged reference state. In this case, the state can be changed even though the complexity of formation is zero. However, the original CA complexity also changes abruptly at the time $\Delta t_{c}[23]$. For better understanding of this abrupt change, it will be important to understand the boundary field theory interpretation of $\Delta t_{c}$.
} 
At the region II, the coordinate $r$ can run from $r_{0}$ to $r_{h}$. For every given $r$, the upper and inferior limits for $t$ in the integration (3.21) are given by the line equations $A B$ and $B C$, respectively. Then we have,

$$
I_{\text {bulk,II }}=-\frac{\Sigma_{d-1} d}{8 \pi \ell_{\mathrm{AdS}}^{2}} \int_{r_{0}}^{r_{h}} r^{d-1} \mathrm{~d} r \int_{t_{R}+r^{*}(r)-r_{m}^{*}}^{r_{m}^{*}-r^{*}(r)} \mathrm{d} t .
$$

For the region III and region IV, we have

$$
I_{\text {bulk }, \mathrm{III}}=-\frac{\Sigma_{d-1} d}{8 \pi \ell_{\mathrm{AdS}}^{2}} \int_{r_{h}}^{r_{m}} r^{d-1} \mathrm{~d} r \int_{t_{R}+r^{*}(r)-r_{m}^{*}}^{t_{R}+r_{m}^{*}-r^{*}(r)} \mathrm{d} t=I_{\mathrm{bulk}, \mathrm{I}},
$$

and

$$
I_{\mathrm{bulk}, \mathrm{IV}}=-\frac{\Sigma_{d-1} d}{8 \pi \ell_{\mathrm{AdS}}^{2}} \int_{0}^{r_{h}} r^{d-1} \mathrm{~d} r \int_{r^{*}(r)-r_{m}^{*}}^{t_{R}+r_{m}^{*}-r^{*}(r)} \mathrm{d} t .
$$

Combining eqs. (3.22), (3.23), (3.24) and (3.25) we find that

$$
\begin{aligned}
I_{\mathrm{bulk}}\left(t_{R}\right) & =I_{\mathrm{bulk}, \mathrm{I}}+I_{\mathrm{bulk}, \mathrm{II}}+I_{\mathrm{bulk}, \mathrm{III}}+I_{\mathrm{bulk}, \mathrm{IV}} \\
& =I_{\mathrm{bulk}, 0}+\frac{\Sigma_{d-1} d}{8 \pi \ell_{\mathrm{AdS}}^{2}} \int_{0}^{r_{0}} r^{d-1} \mathrm{~d} r \int_{t_{R}+r^{*}(r)-r_{m}^{*}}^{r_{m}^{*}-r^{*}(r)} \mathrm{d} t \\
& =I_{\mathrm{bulk}, 0}+\frac{\Sigma_{d-1}}{4 \pi \ell_{\mathrm{AdS}}^{2}}\left[\left.r^{d}\left(r_{m}^{*}-r^{*}-\frac{t_{R}}{2}\right)\right|_{0} ^{r_{0}}+\int_{0}^{r_{0}} r^{d-2} f^{-1}(r) \mathrm{d} r\right] \\
& =I_{\mathrm{bulk}, 0}+\frac{\Sigma_{d-1}}{4 \pi \ell_{\mathrm{AdS}}^{2}} \int_{0}^{r_{0}} r^{d-2} f^{-1}(r) \mathrm{d} r .
\end{aligned}
$$

Here $I_{\text {bulk, } 0}$ is the bulk on-shell action in the case that $t_{R}=0$.

Let us turn to the boundary terms. There are four null boundaries and a spacelike boundary. As the normal vector fields are affinely parameterized, the only possible boundary terms for the null boundaries are the integration of $L_{0}$. Based on the results in the ref. [30], we see that

$$
\begin{aligned}
I_{\mathcal{N}}\left(t_{R}\right) & =I_{\mathcal{N}, 0}+\frac{(d-1) \Sigma_{d-1}}{4 \pi} \int_{r_{0}}^{0} r^{d-2}\left\{1+\ln \left[\frac{(d-1) \ell_{\mathrm{AdS}}}{r}\right]\right\} \mathrm{d} r \\
& =I_{\mathcal{N}, 0}-\frac{\Sigma_{d-1}}{4 \pi}\left\{\ln \left[\frac{(d-1) \ell_{\mathrm{AdS}}}{r_{0}}\right]+\frac{1}{(d-1)}\right\} r_{0}^{d-1} .
\end{aligned}
$$

Here $I_{\mathcal{N}, 0}$ is the value of $I_{\mathcal{N}}\left(t_{R}\right)$ at $t_{R}=0$. The other boundary term comes from the spacelike boundary $D E$. This boundary term is given by the GHY boundary term which reads

$$
I_{\mathrm{GHY}}\left(t_{R}\right)=\frac{\Sigma_{d-1} r_{h}^{d} d}{16 \pi \ell_{\mathrm{AdS}}^{2}}\left[t_{R}+2 r_{m}^{*}-2 r^{*}(0)\right]
$$

based on ref. [32].

Comparing with the case of $t_{R}=0$, we only have one new additional null-null joint term at $r_{0}$ which is

$$
I_{\text {joints }}\left(t_{R}\right)=I_{\text {joints }, 0}-\frac{r_{0}^{d-1} \Sigma_{d-1}}{8 \pi} \ln \left[-r_{0}^{2} f\left(r_{0}\right)\right],
$$

where $I_{\text {joints }, 0}$ is the value of $I_{\text {joints }}\left(t_{R}\right)$ at $t_{R}=0$. 
Because of the time translation symmetry, the surface counterterms are the same as the cases of $t_{R}=0$. Thus the renormalized holographic complexity potential is

$$
\begin{aligned}
\mathcal{C}_{\mathrm{A}, \text { ren }}\left(T, t_{R}\right)= & \mathcal{C}_{\mathrm{A}, \text { ren }, 0}+\frac{\Sigma_{d-1}}{4 \pi^{2} \hbar}\left\{\ell_{\mathrm{AdS}}^{-2} \int_{0}^{r_{0}} r^{d-2} f^{-1}(r) \mathrm{d} r+\frac{r_{h}^{d}\left(t_{R}-\Delta t_{c}\right) d}{4 \ell_{\mathrm{AdS}}^{2}}\right. \\
& \left.-\frac{r_{0}^{d-1}}{2}\left[\ln \left(-r_{0}^{2} f\left(r_{0}\right)\right)+2 \ln \left(\frac{(d-1) \ell_{\mathrm{AdS}}}{r_{0}}\right)+\frac{2}{d-1}\right]\right\},
\end{aligned}
$$

where $r_{0}$ is the function of $t_{R}$ and defined by the eq. (3.19) with $r_{m} \rightarrow \infty$. The value of $\mathcal{C}_{\mathrm{A}, \text { ren }, 0}$ is given by eq. (3.17).

\subsubsection{Time dependnent complexity}

For convenience we choose $y=r_{0} / r_{h}$ and $x=\left(r_{h} / \ell_{\mathrm{AdS}}\right)^{d-1}$ as two free parameters of complexity potential. In this new coordinate, the state $\left|\operatorname{TFD}\left(T, t_{R}\right)\right\rangle$ is $|\operatorname{TFD}(x, y)\rangle$. Let us define a dimensionless renormalized complexity potential $G(x, y)$ such that,

$$
\mathcal{C}_{\mathrm{A}, \mathrm{ren}}(x, y)=\frac{\ell_{\mathrm{AdS}}^{d-1} \Sigma_{d-1}}{4 \pi^{2} \hbar} G(x, y)
$$

Comparing with eq. (3.30) we find

$$
G(x, y)=x^{-1}\left[\pi \frac{d-2}{d} \cot \frac{\pi}{d}+h(y)\right],
$$

with

$$
h(y)=\int_{0}^{y} s^{d-2} f_{1}^{-1}(s) \mathrm{d} s+\frac{f_{2}(y) d}{2}-\frac{y^{d-1}}{2}\left[\ln \left(-f_{1}(y)\right)+2 \ln (d-1)+\frac{2}{d-1}\right],
$$

and

$$
f_{1}(y)=1-\frac{1}{y^{d}}, \quad f_{2}(y)=\frac{\pi}{d} \cot \frac{\pi}{d}-\frac{1}{y}\left[{ }_{2} F_{1}\left(1,-1 / d ; 1-1 / d ; y^{d}\right)-1\right] .
$$

When $d \geq 3$, the vacuum state is given by the parameter $x \rightarrow 0, y=0$. Suppose that $x=x_{0}$ and $y=y_{0}$ stand for an arbitrary TFD state. According to eq. (2.9), the minimal length connecting $(0,0)$ and $\left(x_{0}, y_{0}\right)$ is given by $\left|\mathcal{C}_{\mathrm{A}, \text { ren }}(0,0)-\mathcal{C}_{\mathrm{A}, \text { ren }}\left(x_{0}, y_{0}\right)\right|=\left|\mathcal{C}_{\mathrm{A} \text {,ren }}\left(x_{0}, y_{0}\right)\right|$. However, when $d=2$, as ref. [29] suggested, the corresponding vacuum state is not the one of $r_{h}=0$. Instead, the vacuum state is given by $f(r)=1 / \ell_{\mathrm{AdS}}^{2}+1 / r^{2}$. The renormalized holographic complexity potential for this vacuum state is

$$
\frac{\pi \hbar \mathcal{C}_{\mathrm{A}, \mathrm{BTZ}, \mathrm{vac}}}{\Sigma_{1}}=-\frac{\pi \ell_{\mathrm{AdS}}}{2}
$$

Finally, we obtain the following complexity between the TFD state and its vacuum state

$$
\begin{aligned}
\mathcal{C}\left(\left|\operatorname{TFD}\left(T, t_{R}\right)\right\rangle,|0\rangle\right) & =\frac{r_{h}^{d-1} \Sigma_{d-1}}{4 \pi^{2} \hbar}\left[G\left(x_{0}, y_{0}\right)+2 \pi^{2} \delta_{2, d}\right] \\
& =\frac{d}{\pi^{2}(d-1)}\left[G\left(x_{0}, y_{0}\right)+2 \pi^{2} \delta_{2, d}\right] \frac{M}{\hbar T},
\end{aligned}
$$



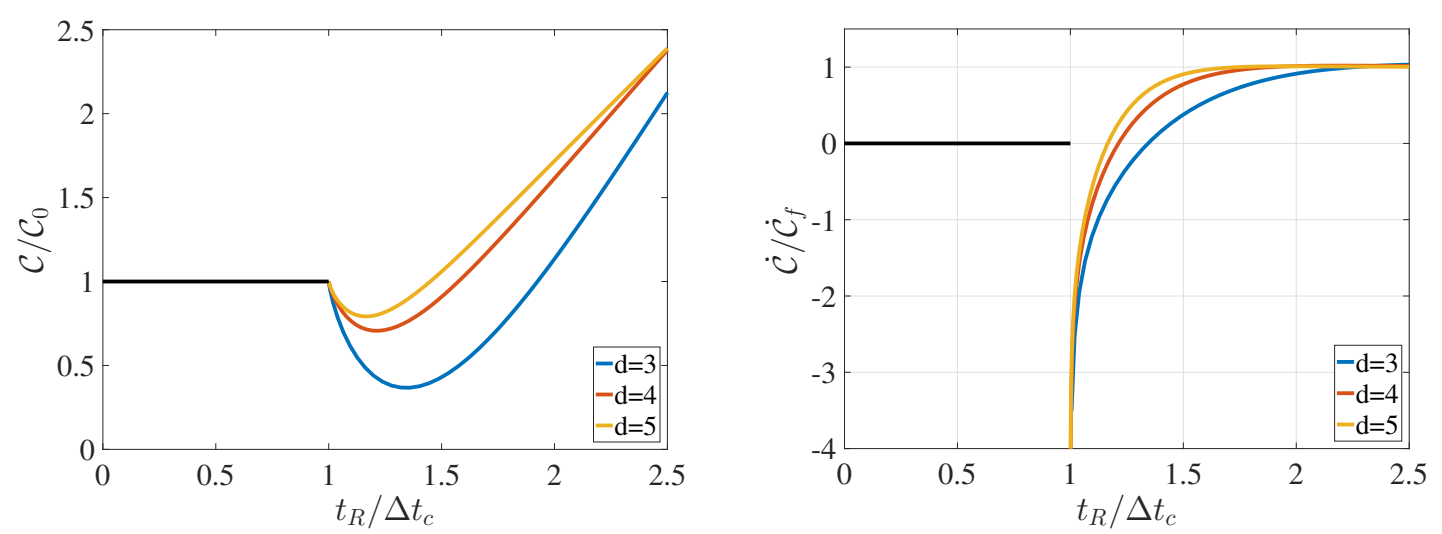

Figure 2. The complexity $\mathcal{C}\left(\left|\operatorname{TFD}\left(0, t_{R}\right)\right\rangle,|0\rangle\right)$ and its growth rate when $d>2$. $\mathcal{C}_{0}$ is the complexity when $t_{R}=\Delta t_{c}$ and $\dot{\mathcal{C}}_{f}=2 M / \pi \hbar$. Higher dimensional cases give the similar results.

with the relationships $T=x_{0}^{1 /(d-1)} d /\left(4 \pi \ell_{\mathrm{AdS}}\right)$ and $t_{R}=2 \ell_{\mathrm{AdS}} x^{-1 /(d-1)} f_{2}\left(y_{0}\right)$. The absolute symbol has been dropped because the right side of eq. (3.36) is always positive when $d>2$ (we confirmed it numerically from $d=2$ to $d=10$.). The growth rate can be obtained directly from this expression, which reads ${ }^{10}$

$$
\begin{aligned}
& \frac{\mathrm{d}}{\mathrm{d} t_{R}} \mathcal{C}\left(\left|\operatorname{TFD}\left(T, t_{R}\right)\right\rangle,|0\rangle\right) \\
& \quad=\frac{2 M}{\pi \hbar}\left\{1+\frac{y_{0}^{d} f_{1}\left(y_{0}\right)}{2 \pi \hbar}\left[\ln \left(-f_{1}\left(y_{0}\right)\right)+2 \ln (d-1)\right)\right\} .
\end{aligned}
$$

The time evolution of the complexity $\mathcal{C}\left(\left|\operatorname{TFD}\left(T, t_{R}\right)\right\rangle,|0\rangle\right)$ and its growth rate are shown in figure 2 and figure 3 . We find that the relationship between the complexity and $t_{R}$ is not monotonic. When $t_{R}$ runs from $\Delta t_{c}$ to infinite, the value of complexity will first decrease and then increase, so there is a minimal value. For the case that $t_{R} \rightarrow \Delta t_{c}$, we have $y_{0} \rightarrow 0^{+}$. Thus eq. (3.37) shows that

$$
\frac{\mathrm{d}}{\mathrm{d} t_{R}} \mathcal{C}\left(\left|\operatorname{TFD}\left(T, t_{R}\right)\right\rangle,|0\rangle\right) \rightarrow-\infty, \quad \text { as } t_{R} \rightarrow \Delta t_{c} .
$$

In the late limit $t_{R} \rightarrow \infty$, it saturates to the Lloyd's bound,

$$
\lim _{t_{R} \rightarrow \infty} \frac{\mathrm{d}}{\mathrm{d} t_{R}} \mathcal{C}\left(\left|\operatorname{TFD}\left(0, t_{R}\right)\right\rangle,|0\rangle\right)=\lim _{t_{R} \rightarrow \infty} \dot{\mathcal{C}}_{\mathrm{A}, \text { ren }}=\lim _{y_{0} \rightarrow 1} \dot{\mathcal{C}}_{\mathrm{A}, \text { ren }}=\frac{2 M}{\pi \hbar} .
$$

From figure 3 it is clear that the Lloyd's bound is violated in the intermediate and large time for the BTZ black hole $(d=2)$, but it is not so clear if this is the case also for $d>2$ from figure 2. To check it we consider the the subleading term from eq. (3.37) in the late time limit:

$$
\frac{\mathrm{d}}{\mathrm{d} t_{R}} \mathcal{C}\left(\left|\operatorname{TFD}\left(T, t_{R}\right)\right\rangle,|0\rangle\right)-\frac{2 M}{\pi \hbar}=\frac{2 M}{\pi \hbar} \frac{y_{0}^{d} f_{1}\left(y_{0}\right)}{2 \pi \hbar} \ln \left[-f_{1}\left(y_{0}\right)\right]+\mathcal{O}\left(y_{0}-1\right) .
$$

\footnotetext{
${ }^{10}$ When this paper was finished, ref. [23] appeared which also studied the complexity growth rate. Our result eq. (3.37) is the same as eq. (E.9) in ref. [23].
} 

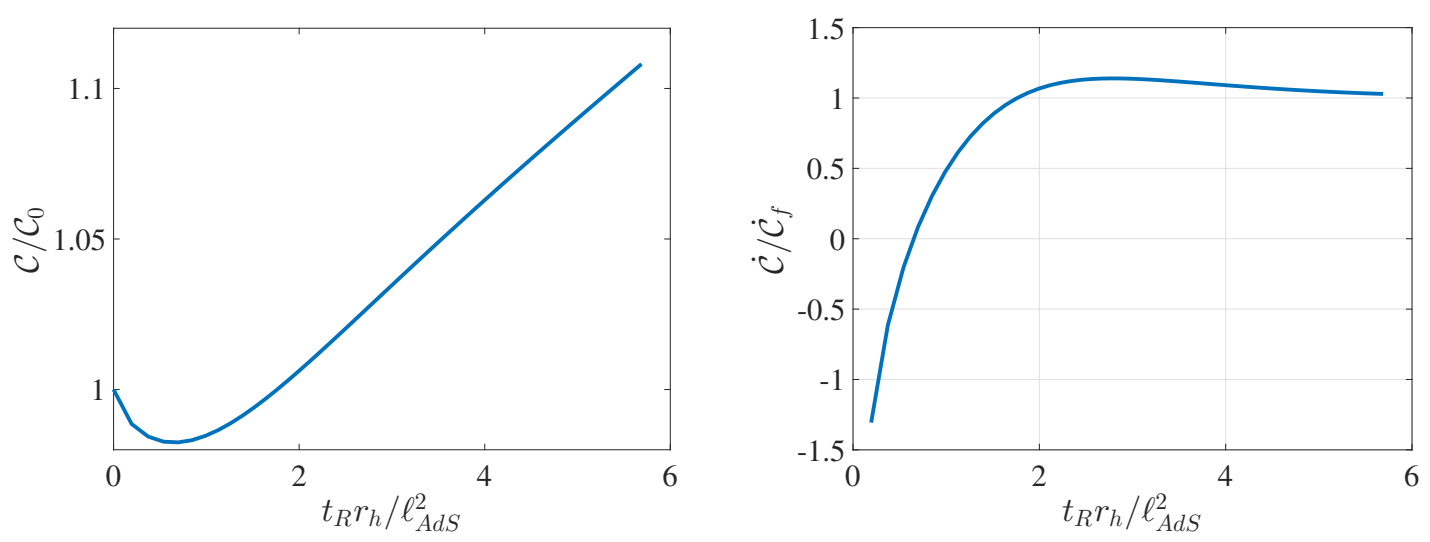

Figure 3. The complexity $\mathcal{C}\left(\left|\operatorname{TFD}\left(0, t_{R}\right)\right\rangle,|0\rangle\right)$ and their growth rates for the BTZ black hole. $\mathcal{C}_{0}$ is the complexity when $t_{R}=0$ and $\dot{\mathcal{C}}_{f}=2 M / \pi \hbar$.

As $y_{0} \in(0,1), f_{1}\left(y_{0}\right)=1-1 / y_{0}^{d}<0$. In the late time limit, $y_{0} \rightarrow 1^{-}$and the first term in the right-side of eq. (3.40) dominant, which means that the subleading term is positive. Thus the CA conjecture violates the Lloyd's bound slightly in the large time.

\subsection{CV conjecture}

In this subsection, we compute the time-dependent complexity of the $\mathrm{AdS}_{d+1}$ Schwarzschild planar black holes in the CV conjecture. Let us rewrite the metric (3.7) in the following form

$$
\mathrm{d} s^{2}=\ell_{\text {AdS }}^{2}\left(-g^{2}(\rho) \mathrm{d} \tilde{t}^{2}+\mathrm{d} \rho^{2}+h^{2}(\rho) \sum_{i=1}^{d-1} \mathrm{~d} \tilde{x}_{i}^{2}\right),
$$

where

$$
h(\rho)=\left(\cosh \frac{d \rho}{2}\right)^{2 / d}, g(\rho)=h(\rho) \tanh \frac{d \rho}{2} .
$$

Here we introduced dimensionless variables $\tilde{t}=\frac{r_{h}}{\ell_{\mathrm{AdS}}^{2}} t, \tilde{x}_{i}=\frac{r_{h}}{\ell_{\mathrm{AdS}}^{2}} x_{i}, \tilde{r}=\frac{r}{r_{h}}$ and performed a coordinate transformation $\mathrm{d} \rho=\frac{\mathrm{d} \tilde{r}}{\tilde{r} \sqrt{1-1 / \tilde{r}}}$. The Penrose diagram is shown in figure 4 . Similarly to the CA case, the renormalized complexity potential only depends on $t_{L}+t_{R}$.

We can continue (3.41) into the interior region of figure 4 by setting $\rho=i \kappa$ and $\tilde{t}_{I}=\tilde{t}+i \frac{\pi}{2}$. For the case $\tilde{t}_{B} \equiv \tilde{t}_{R}=\tilde{t}_{L}$, the maximal volume surface is given by the blue line in figure 4 . The upper red dotted line is for $\tilde{t}_{B}=\infty$ and the middle red dotted line is for $\tilde{t}_{B}=0$. The corresponding volume of this codimension-one surface is described by the following integration

$$
V=\tilde{\Sigma}_{d-1} \ell_{\text {AdS }}^{d} \int h(\rho)^{d-1} \sqrt{-g^{2}(\rho)+(\partial \rho / \partial \tilde{t})^{2}} \mathrm{~d} \tilde{t},
$$

where $\tilde{\Sigma}_{d-1} \equiv \int d^{d-1} \tilde{x}$ is the volume of the spatial geometry. The volume can be maximized following [37-39].

In principle, we should solve the Euler-Lagrangian equation of (3.43) to find $\rho(\tilde{t})$. Alternatively, following [37] we may find the first integral of the equation of motion of (3.43). 


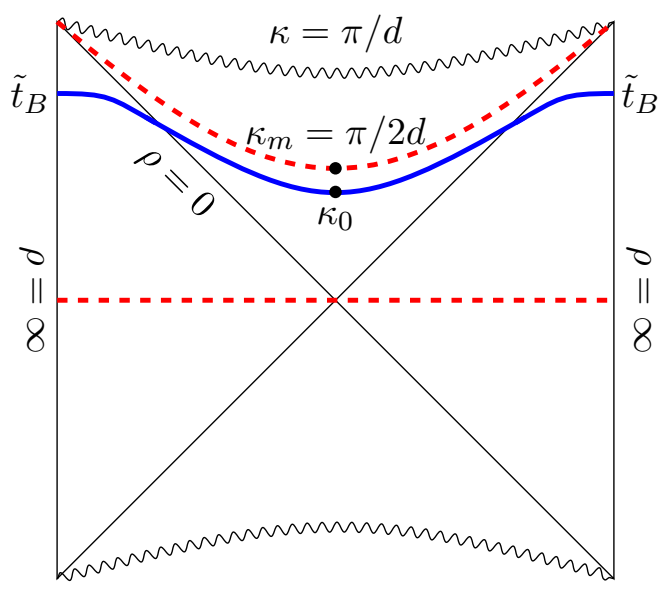

Figure 4. Extremal surfaces in the AdS black hole. For given time slices at the left and right boundary, the volume of extremal surface connecting these two time slices (blue curve) gives the holographic complexity potential. The upper red dotted line is for $\tilde{t}_{B}=\infty$ and the middle red dotted line is for $\tilde{t}_{B}=0$.

In other words, because the integrand of (3.43) is time independent the Hamiltonian is conserved:

$$
\mathcal{H}=\frac{\partial \mathcal{L}}{\partial \rho^{\prime}(\tilde{t})}-\mathcal{L}=\text { const. }
$$

which yields

$$
\frac{g^{2} h^{d-1}}{\sqrt{-g^{2}+(\partial \rho / \partial \tilde{t})^{2}}}=i g_{0} h_{0}^{d-1},
$$

where $h_{0}:=h\left(i \kappa_{0}\right)$ and $g_{0}:=g\left(i \kappa_{0}\right)$ with $\kappa_{0}\left(0<\kappa_{0}<\frac{\pi}{2 d}\right)$ satisfying $\left.\frac{\partial \kappa}{\partial \tilde{t}}\right|_{\kappa=\kappa_{0}}=0$. From (3.45), we can write the time $\tilde{t}_{B}$ in terms of $\kappa_{0}$

$$
\begin{aligned}
\tilde{t}_{B}= & \int_{\epsilon}^{\kappa_{0}} \frac{\mathrm{d} \kappa}{\left(\cos \frac{d \kappa}{2}\right)^{\frac{2}{d}} \tan \frac{d \kappa}{2} \sqrt{1-\frac{\sin ^{2} d \kappa}{\sin ^{2} d \kappa_{0}}}}-\int_{\epsilon}^{\infty} \frac{\mathrm{d} \rho}{\left(\cosh \frac{d \rho}{2}\right)^{\frac{2}{d}} \tanh \frac{d \rho}{2} \sqrt{1+\frac{\sinh ^{2} d \rho}{\sin ^{2} d \kappa_{0}}}} \\
= & \int_{0}^{\kappa_{0}}\left(\frac{\left(\cos \frac{d \kappa}{2}\right)^{-\frac{2}{d}} \cot \frac{d \kappa}{2}}{\sqrt{1-\csc ^{2} d \kappa_{0} \sin ^{2} d \kappa}}-\frac{\left(\cosh \frac{d \kappa}{2}\right)^{-\frac{2}{d}} \operatorname{coth} \frac{d \kappa}{2}}{\sqrt{1+\csc ^{2} d \kappa_{0} \sinh ^{2} d \kappa}}\right) \mathrm{d} \kappa \\
& -\int_{\kappa_{0}}^{\infty} \frac{\left(\cosh \frac{d \rho}{2}\right)^{-\frac{2}{d}} \operatorname{coth} \frac{d \rho}{2}}{\sqrt{1+\csc ^{2} d \kappa_{0} \sinh ^{2} d \rho}} \mathrm{d} \rho .
\end{aligned}
$$

Substituting (3.45) into (3.43), the maximum volume can be expressed in terms of the parameter $\kappa_{0}$,

$$
V=2 \tilde{\Sigma}_{d-1} \ell_{\text {AdS }}^{d}\left(\int_{0}^{\kappa_{0}} \frac{\left(\cos \frac{d \kappa}{2}\right)^{\frac{2(d-1)}{d}}}{\sqrt{\frac{\sin ^{2} d \kappa_{0}}{\sin ^{2} d \kappa}-1}} \mathrm{~d} \kappa+\int_{0}^{\rho_{\infty}} \frac{\left(\cosh \frac{d \rho}{2}\right)^{\frac{2(d-1)}{d}}}{\sqrt{1+\frac{\sin ^{2} d \kappa_{0}}{\sinh ^{2} d \rho}}} \mathrm{d} \rho\right) .
$$


Here we have introduced the UV cut off $\rho_{\infty}$, IR cut off $\epsilon$ and the factor 2 comes from the symmetry of figure 4 .

To evaluate the renormalized holographic complexity potantial, we will subtract the surface counterterms which were obtained for $d \geq 2$ in ref. [30]:

$$
\begin{aligned}
V_{\mathrm{ct}}^{(1)} & =\frac{\ell_{\mathrm{AdS}}}{d-1} \int_{B} d^{d-1} \tilde{x} \sqrt{\sigma}=\frac{\tilde{\Sigma}_{d-1} \ell_{\mathrm{AdS}}^{d}}{d-1}\left(\cosh \frac{d \rho_{\infty}}{2}\right)^{2(d-1) / d}, \\
V_{\mathrm{ct}}^{(n)} & =0, \quad n>1,
\end{aligned}
$$

where $\sigma$ is the induced metric of the time slice on the boundary. Hence the renormalized holographic complexity potential can be written as

$$
\begin{aligned}
\mathcal{C}_{\mathrm{V}, \text { ren }} & =\frac{1}{\ell} \lim _{\delta \rightarrow 0}\left(V-2 V_{\mathrm{ct}}^{(1)}\right) \\
& =\frac{2 \tilde{\Sigma}_{d-1} \ell_{\text {AdS }}^{d}}{\ell}\left(\int_{0}^{\kappa_{0}} \frac{\left(\cos \frac{d \kappa}{2}\right)^{\frac{2(d-1)}{d}}}{\sqrt{\frac{\sin ^{2} d \kappa_{0}}{\sin ^{2} d \kappa}-1}} \mathrm{~d} \kappa+\int_{0}^{\infty}\left(\frac{\left(\cosh \frac{d \rho}{2}\right)^{\frac{2(d-1)}{d}}}{\sqrt{1+\frac{\sin ^{2} d \kappa_{0}}{\sinh ^{2} d \rho}}}-\frac{\cosh \frac{d \rho}{2}}{\left(\sinh \frac{d \rho}{2}\right)^{\frac{d}{d-2}}}\right) \mathrm{d} \rho\right) .
\end{aligned}
$$

As in the CA conjecture, the renormalized holographic complexity potential of Schwarzschild AdS black holes at the zero temperature limit are all zeros, the complexity between $\left|\operatorname{TFD}\left(T, t_{L}+t_{R}\right)\right\rangle$ and $|\operatorname{TFD}(0,0)\rangle$ then is ${ }^{11}$

$$
\mathcal{C}\left(\left|\operatorname{TFD}\left(T, t_{L}+t_{R}\right)\right\rangle,|0\rangle\right)=\mathcal{C}_{\mathrm{V}, \mathrm{ren}}
$$

However, for the BTZ black hole, the vacuum state is not the one of zero horizon. We have to choose the solution $f(r)=1 / \ell_{\mathrm{AdS}}^{2}+1 / r^{2}$ for the vacuum state. Then the renormalized holographic complexity potential of this vacuum state is given by $\mathcal{C}_{V, \mathrm{BTZ} \text {,vac }}=-4 \pi \ell_{\text {AdS }}^{2} / \ell$. Thus we have the following complexity for the BTZ black hole

$$
\mathcal{C}\left(\left|\operatorname{TFD}\left(T, t_{L}+t_{R}\right)\right\rangle,|0\rangle\right)=\mathcal{C}_{\mathrm{V}, \mathrm{ren}}+4 \pi \ell_{\mathrm{AdS}}^{2} / \ell .
$$

Combining eqs. (3.50) and (3.51) we have an expression

$$
\mathcal{C}\left(\left|\operatorname{TFD}\left(T, t_{L}+t_{R}\right)\right\rangle,|0\rangle\right)=\mathcal{C}_{\mathrm{V}, \mathrm{ren}}+4 \pi \delta_{2, d} \ell_{\text {AdS }}^{2} / \ell .
$$

The time evolution of the complexity $\mathcal{C}\left(\left|\operatorname{TFD}\left(T, t_{L}+t_{R}\right)\right\rangle,|0\rangle\right)$ and its growth rate are shown in figure 5 where the relationship between the complexity and $t_{L}+t_{R}$ is monotonic contrary to the CA case.

In the early time limit $\left(\tilde{t}_{B} \rightarrow 0\right.$ or $\left.\kappa_{0} \rightarrow 0\right)$, we have

$$
\tilde{t}_{B}=-\frac{\sin d \kappa_{0}}{2} \int_{0}^{\rho_{\infty}} \frac{\mathrm{d} \rho}{\left(\cosh \frac{d \rho}{2}\right)^{\frac{2}{d}} \sinh ^{2} \frac{d \rho}{2}},
$$

\footnotetext{
${ }^{11}$ We have substituted $t_{L}+t_{R}$ for $2 t_{B}$ for comparison with the results obtained by other methods.
} 

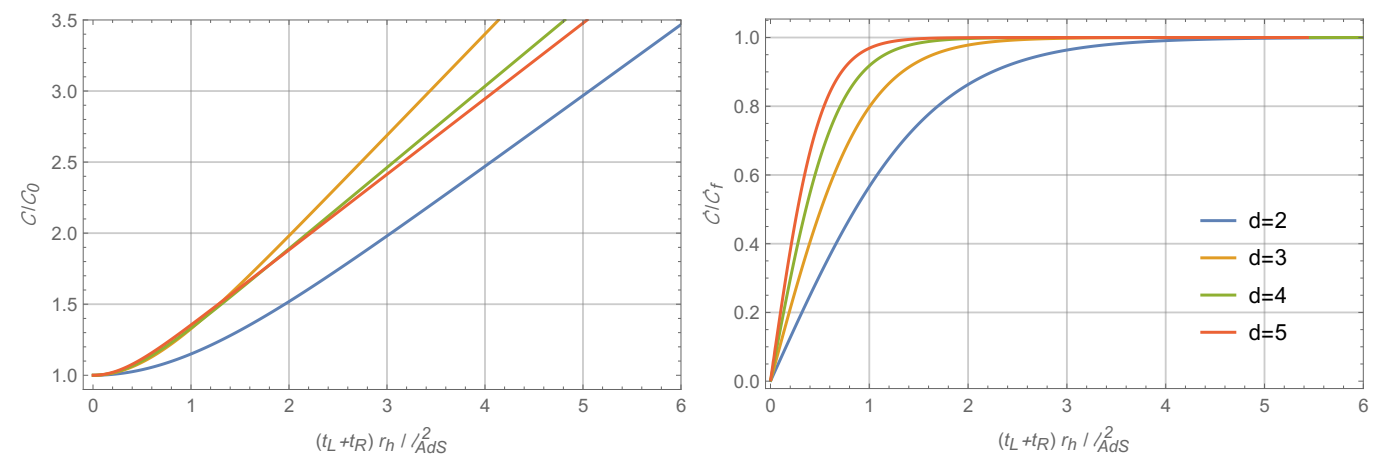

Figure 5. The values of $\mathcal{C}\left(\left|\operatorname{TFD}\left(T, t_{L}+t_{R}\right)\right\rangle,|0\rangle\right)$ and its growth rate when $d=2,3,4,5$. The higher dimensions give similar results. $\mathcal{C}_{0}$ is the complexity when $t_{L}+t_{R}=0$ and $\dot{\mathcal{C}}_{f}$ is the Lioyd's bound of growth rate, which is given by eq. (3.58).

so the complexity $\mathcal{C}\left(\left|\operatorname{TFD}\left(T, t_{L}+t_{R}\right)\right\rangle,|0\rangle\right)$ can be written as

$$
\begin{aligned}
& \mathcal{C}\left(\left|\operatorname{TFD}\left(T, t_{L}+t_{R}\right)\right\rangle,|0\rangle\right)=\mathcal{C}_{\mathrm{V}, \mathrm{ren}}+4 \pi \delta_{2, d} \ell_{\mathrm{AdS}}^{2} \\
& \quad=\frac{\tilde{\Sigma}_{d-1} \ell_{\mathrm{AdS}}^{d}}{\ell}\left(\frac{\sqrt{\pi}(d-2) \Gamma\left(1+\frac{1}{d}\right)}{(d-1) \Gamma\left(\frac{1}{2}+\frac{1}{d}\right)}+\frac{r_{h}^{2} d^{2} \Gamma\left(\frac{1}{2}+\frac{1}{d}\right)}{8 \ell_{\mathrm{AdS}}^{4} \sqrt{\pi} \Gamma\left(\frac{1}{d}\right)}\left(t_{L}+t_{R}\right)^{2}+\ldots\right)+4 \pi \delta_{2, d} \ell_{\mathrm{AdS}}^{2} / \ell .
\end{aligned}
$$

At $t_{L}+t_{R}=0$,

$$
\mathcal{C}(|\operatorname{TFD}(T, 0)\rangle,|0\rangle)=\frac{d \sqrt{\pi}(d-2) \Gamma\left(1+\frac{1}{d}\right)}{\pi^{2}(d-1) \Gamma\left(\frac{1}{2}+\frac{1}{d}\right)} \frac{M}{\hbar T}+\frac{(d-1) \ell_{\mathrm{AdS}}}{\pi \hbar} \delta_{2, d},
$$

where we use eq. (3.9) and take the length scale $\ell / \ell_{\text {AdS }}=4 \pi^{2} \hbar /(d-1)$.

In the late time limit $\left(\tilde{t}_{B} \rightarrow \infty\right.$ or $\left.\kappa_{0} \rightarrow \kappa_{m}=\frac{\pi}{2 d}\right)$, the renormalized complexity (3.49) becomes

$$
\begin{aligned}
\mathcal{C}\left(\left|\operatorname{TFD}\left(T, t_{L}+t_{R}\right)\right\rangle,|0\rangle\right)=\mathcal{C}_{\mathrm{V}, \text { ren }} \\
=\frac{\tilde{\Sigma}_{d-1} \ell_{\text {AdS }}^{d}}{\ell}\left(\frac{r_{h}}{2 \ell_{\mathrm{AdS}}^{2}}\left(t_{L}+t_{R}\right)-\int_{\epsilon}^{\frac{\pi}{2 d}} \frac{\cos d \kappa \mathrm{d} \kappa}{\left(\cos \frac{d \kappa}{2}\right)^{2 / d} \tan \frac{d \kappa}{2}}+\int_{\epsilon}^{\rho_{\infty}} \frac{\operatorname{coth} \frac{d \rho}{2} \mathrm{~d} \rho}{\left(\cosh \frac{d \rho}{2}\right)^{2 / d}}-\frac{2}{d-1}\right) \\
=\frac{\tilde{\Sigma}_{d-1} \ell_{\text {AdS }}^{d}}{\ell}\left(\frac{r_{h}}{2 \ell_{\mathrm{AdS}}^{2}}\left(t_{L}+t_{R}\right)+\text { finite term }\right) .
\end{aligned}
$$

Thus the complexity growth rate in the late time limit is given by

$$
\lim _{t_{L}+t_{R} \rightarrow \infty} \frac{\mathrm{d}}{\mathrm{d}\left(t_{L}+t_{R}\right)} \mathcal{C}\left(\left|\operatorname{TFD}\left(T, t_{L}+t_{R}\right)\right\rangle,|0\rangle\right)=\frac{8 \pi \ell_{\mathrm{AdS}} M}{\ell(d-1)},
$$

where we use eq. (3.9). If we take the length scale $\ell / \ell_{\mathrm{AdS}}=4 \pi^{2} \hbar /(d-1)$ we find that the Lloyd's bound is satisfied in the CV conjecture:

$$
\frac{\mathrm{d}}{\mathrm{d}\left(t_{L}+t_{R}\right)} \mathcal{C}\left(\left|\operatorname{TFD}\left(T, t_{L}+t_{R}\right)\right\rangle,|0\rangle\right)<\frac{2 M}{\pi \hbar} .
$$




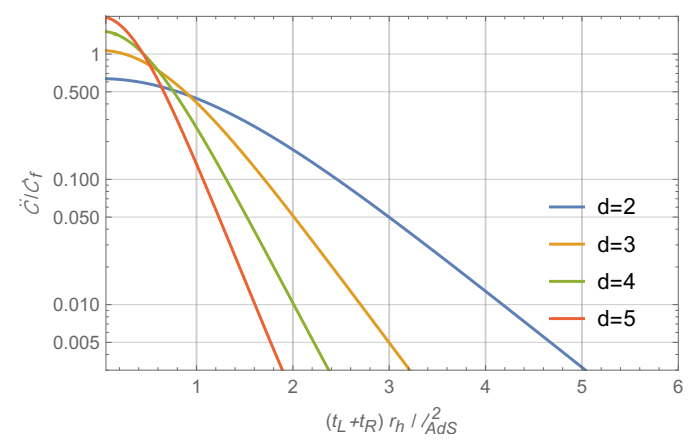

Figure 6. The values of $\ddot{\mathcal{C}}$ for $d=2,3,4,5 . \dot{\mathcal{C}}_{f}$ is the Lloyd's bound of growth rate, which is given by eq. (3.58). We can see that $\ddot{\mathcal{C}}>0$ and so $\dot{\mathcal{C}}_{f}$ is the maximum value of $\dot{\mathcal{C}}$.

Numerical results show that this is the maximum value of the growth rate at all time (see figure 6), which is different from the CA conjecture.

Let us make a comparison for the complexity growth rates between the CA and CV conjectures. From figures 2, 3 and 5 we see that at early time, two conjectures give different results. In the CA conjecture, we see that the complexity between the TFD state and the vacuum state does not change until $t_{R}+t_{L}=\Delta t_{c}$ for $d>2$. When $t>\Delta t_{c}$, the CA conjecture predicts the complexity will decrease first and then increase. The growth rate at $t=\Delta t_{c}$ is negative infinite. In the $\mathrm{CV}$ conjecture, we see that the complexity between the TFD state and the vacuum state always increase with the order of $t^{2}$ when $t$ is small. In the late time limit, two conjectures predict the complexity will increase linearly in $t$ and the slope is proportional to total mass $M$. However, in the large time region, the CA conjecture will approach to $2 M$ from a larger value, so it violates the Lloyd's bound. If we choose the length scale $\ell=4 \pi^{2} \hbar \ell_{\mathrm{AdS}} /(d-1)$, the CV conjecture satisfies the Lloyd's bound at all time and saturates to the Lloyd's bound in the late time limit.

\section{Time dependent complexity of the TFD states: field theory approach}

In this section we compute the complexity by the field theoretic methods proposed by refs. $[15,16]$. One is the FS method [15] based on the Fubini-Study metric and the other is the FG method [16] based on the Finsler geometry. As a common basis of two methods we start with constructing a time-dependent TFD state for free field theory explicitly.

\subsection{Time evolution of the TFD states}

Both in the FS and FG methods, a crucial step is to find the transformation from vacuum state to a TFD state. We will follow the method proposed by ref. [16]. Let us consider a bosonic Hilbert space $\mathcal{H}$ and the occupation number representation. Suppose that $\hat{a}_{\vec{k}_{i}}$ and $\hat{a}_{\vec{k}_{i}}^{\dagger}$ are the annihilation and creation operators, which can annihilate or create a particle of momentum $\vec{k}_{i}$. The particle number density operator at momentum $\vec{k}_{i}$ is defined as

$$
\hat{N}_{\vec{k}_{i}}:=\hat{a}_{\vec{k}_{i}}^{\dagger} \hat{a}_{\vec{k}_{i}} \cdot
$$


As the particle number density operators for different momentum commute each other, their common eigenstates form a complete basis in the Hilbert space $\mathcal{H}$. Let us assume the momentum is discrete and introduce the notation,

$$
\prod_{i}\left|n_{i}, \vec{k}_{i}\right\rangle:=\left|n_{0}, \vec{k}_{0}\right\rangle\left|n_{1}, \vec{k}_{1}\right\rangle\left|n_{2}, \vec{k}_{2}\right\rangle \cdots
$$

to stand for one common eigenstate for all the particle number operators. Here the product includes all the possible momentum values. Then any state $|\psi\rangle \in \mathcal{H}$ can be written in the following form

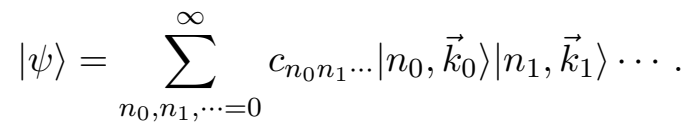

Following a standard procedure of the thermofield double formalism we double the degrees of freedom (the subscript $L$ for one and $R$ for the other) and consider the product space of the two systems as

$$
\left|n, \vec{k}_{i}\right\rangle_{L}\left|n, \vec{k}_{j}\right\rangle_{R}
$$

The thermal vacuum TFD state for the free bosons can be expanded as

$$
|\mathrm{TFD}\rangle=\frac{1}{\sqrt{Z}} \prod_{\vec{k}_{i}} \sum_{n=0}^{\infty} e^{-n \omega_{\vec{k}_{i}} / 2 T}\left|n, \vec{k}_{i}\right\rangle_{L}\left|n, \vec{k}_{i}\right\rangle_{R}
$$

It can be confirmed by computing the reduced density matrix of the total density matrix $\left(\hat{\rho}_{\text {total }}=|\mathrm{TFD}\rangle\langle\mathrm{TFD}|\right)$ in the projected Hilbert space $\mathcal{H}_{L}$ or $\mathcal{H}_{R}$.

$$
\hat{\rho}_{L / R}=\operatorname{tr}_{R / L} \hat{\rho}_{\text {total }}=\frac{1}{Z} \prod_{\vec{k}_{i}} \sum_{n=0}^{\infty} \exp \left(-n \omega_{\vec{k}_{i}} / T\right)\left|n, \vec{k}_{i}\right\rangle\left\langle n, \vec{k}_{i}\right|,
$$

where the factor $1 / Z$ insures that $\operatorname{Tr} \hat{\rho}_{L}=\operatorname{Tr} \hat{\rho}_{R}=1$. This is the density matrix for the system of free bosons with temperature $T$.

The time dependent TFD state is given by eq. (1.2) so we have

$$
\left|\operatorname{TFD}\left(t_{L}, t_{R}\right)\right\rangle=\exp \left[-i\left(\hat{H}_{L} t_{L}+\hat{H}_{R} t_{R}\right)\right]|\mathrm{TFD}\rangle \propto \exp \left[-i\left(\hat{H}_{L} t_{L}+\hat{H}_{R} t_{R}\right)\right] \hat{U}_{T}^{\dagger}|0\rangle,
$$

where

$$
\hat{U}_{T}^{\dagger}:=\prod_{\vec{k}_{i}} \exp \left(e^{-\omega_{\vec{k}_{i}} / 2 T} \hat{a}_{\vec{k}_{i}}^{R \dagger} \hat{a}_{\vec{k}_{i}}^{L \dagger}\right)=\exp \left[\int \mathrm{d}^{d-1} k e^{-\omega_{\vec{k}} / 2 T} \hat{a}^{R \dagger}(\vec{k}) \hat{a}^{L \dagger}(\vec{k})\right],
$$

which can convert the vacuum state $|0\rangle \equiv|0\rangle_{L}|0\rangle_{R}$ into the $|\mathrm{TFD}\rangle$, i.e., $|\mathrm{TFD}\rangle \propto \hat{U}_{T}^{\dagger}|0\rangle$. This transformation can be understood as a Bogoliubov transformation which is explained in appendix A. In the second equality, the discrete form has been converted into a continuous form. ${ }^{12}$ The Hamiltonian $H_{R}$ and $H_{L}$ depend on the dynamic of dual boundary fields.

\footnotetext{
${ }^{12}$ Ref. [16] proves that $\hat{U}_{T}^{\dagger}$ has a unitary partner,

$$
\hat{G}_{T}:=\exp \left\{\int \operatorname{arctanh} e^{-\omega_{\vec{k}} / 2 T}\left[\hat{a}^{R \dagger}(\vec{k}) \hat{a}^{L \dagger}(\vec{k})-\hat{a}^{R}(\vec{k}) \hat{a}^{L}(\vec{k})\right] \mathrm{d}^{d-1} k\right\},
$$

which can also realize $|\mathrm{TFD}\rangle \propto \hat{G}_{T}|0\rangle$.
} 
For the free bosons, the Hamiltonian can be expressed by the creation and annihilation operators in the following way ${ }^{13}$

$$
\hat{H}_{L} t_{L}+\hat{H}_{R} t_{R}=\int \mathrm{d}^{d-1} k \omega_{\vec{k}}\left(\hat{N}_{\vec{k}}^{R} t_{R}+\hat{N}_{\vec{k}}^{L} t_{L}\right)
$$

Although in general we cannot find a function $f(T, \vec{k})$ such that $\exp \left[-i\left(\hat{H}_{L} t_{L}+\hat{H}_{R} t_{R}\right)\right] \hat{U}_{T}^{\dagger}=$ $\exp \left[\int \mathrm{d}^{d-1} k f(T, \vec{k}) \hat{a}^{R \dagger}(\vec{k}) \hat{a}^{L \dagger}(\vec{k})\right]$, we can find a function $f(T, \vec{k})$ satisfying

$$
\left|\operatorname{TFD}\left(t_{L}, t_{R}\right)\right\rangle \propto \exp \left[\int \mathrm{d}^{d-1} k f(T, \vec{k}) \hat{a}^{R \dagger}(\vec{k}) \hat{a}^{L \dagger}(\vec{k})\right]|0\rangle .
$$

To see this, let us plug eqs. (4.10) and (4.5) into eq. (4.7) in the discrete form. Thus we have

$$
\begin{aligned}
\left|\operatorname{TFD}\left(t_{L}, t_{R}\right)\right\rangle & \propto \prod_{\vec{k}_{i}} \sum_{n=0}^{\infty} e^{-n \omega_{\vec{k}_{i}} / 2 T} \exp \left[-i\left(\hat{N}_{\vec{k}}^{R} t_{R}+\hat{N}_{\vec{k}}^{L} t_{L}\right)\right]\left|n, \vec{k}_{i}\right\rangle_{L}\left|n, \vec{k}_{i}\right\rangle_{R} \\
& \propto \prod_{\vec{k}_{i}} \sum_{n=0}^{\infty} e^{-n \omega_{\vec{k}_{i}}\left[1 / 2 T+i\left(t_{R}+t_{L}\right)\right]}\left|n, \vec{k}_{i}\right\rangle_{L}\left|n, \vec{k}_{i}\right\rangle_{R} .
\end{aligned}
$$

Now converting it into the continuous form, we obtain that

$$
\left|\operatorname{TFD}\left(t_{L}, t_{R}\right)\right\rangle \propto \hat{U}_{a}^{\dagger}\left(t_{L}, t_{R}\right)|0\rangle
$$

with the time dependent non-unitary operator ${ }^{14}$

$$
\hat{U}_{a}^{\dagger}\left(t_{L}, t_{R}\right):=\exp \left[\int \mathrm{d}^{d-1} k e^{-\omega_{\vec{k}}\left[1 / 2 T+i\left(t_{R}+t_{L}\right)\right]} \hat{a}^{R \dagger}(\vec{k}) \hat{a}^{L \dagger}(\vec{k})\right] .
$$

This shows that the function in eq. (4.11) is

$$
f(T, \vec{k})=e^{-\omega_{\vec{k}}\left[1 / 2 T+i\left(t_{R}+t_{L}\right)\right]} .
$$

We see that the time dependent TFD state only depends on $t_{L}+t_{R}$. For later use we also define

$$
r_{\vec{k}}:=\operatorname{arctanh}[f(T, \vec{k})]=\operatorname{arctanh} e^{-\omega_{\vec{k}}\left[1 / 2 T+i\left(t_{R}+t_{L}\right)\right]} .
$$

Eqs. (4.16) and (4.17) will play crucial roles when we compute the complexity growth rate in the next subsections.

\footnotetext{
${ }^{13}$ The zero point energy has been neglected, as it only contribute a constant factor on the state.

${ }^{14}$ Note that $\exp \left[-i\left(\hat{H}_{L} t_{L}+\hat{H}_{R} t_{R}\right)\right] \hat{U}_{T}^{\dagger} \neq \hat{U}_{T}^{\dagger}\left(t_{L}, t_{R}\right)$. The non-unitary operator $\hat{U}_{T}^{\dagger}\left(t_{L}, t_{R}\right)$ has a unitary partner:

$$
\hat{G}_{T}\left(t_{L}, t_{R}\right):=\exp \left\{\int\left[r_{\vec{k}} \hat{a}^{R \dagger}(\vec{k}) \hat{a}^{L \dagger}(\vec{k})-r_{\vec{k}}^{*} \hat{a}^{R}(\vec{k}) \hat{a}^{L}(\vec{k})\right] \mathrm{d}^{d-1} k\right\},
$$

Thus the time evolution of the TFD state can be generated by two ways: $\left|\operatorname{TFD}\left(t_{L}, t_{R}\right)\right\rangle \propto \hat{U}_{T}^{\dagger}\left(t_{L}, t_{R}\right)|0\rangle \propto$ $\hat{G}_{T}\left(t_{L}, t_{R}\right)|0\rangle$.
} 


\subsection{Fubini-Study (FS) metric}

Let us first use the method proposed by ref. [15] to compute the complexity between $\left|\operatorname{TFD}\left(t_{L}, t_{R}\right)\right\rangle$ and the zero temperature limit vacuum state $|0\rangle$. This method is based on the Fubini-Study metric (see the appendix B for some basic introduction and refer to ref. [40] for details) and unitary transformations. For a generator set $E=\left\{M^{1}, M^{2}, \cdots\right\}$, the tangent anti-Hermitian operator $\hat{T}$ can be decomposed as

$$
\hat{T}(s)=\sum_{I} Y_{I}(s) M^{I} .
$$

This tangent operator can generate a unitary operator by a time order exponential map

$$
\hat{O}(s):=\overleftarrow{P} \exp \left[\int_{0}^{s} \hat{T}(\tilde{s}) \mathrm{d} \tilde{s}\right]
$$

where $\overleftarrow{\mathcal{P}}$ denotes a time ordering such that the tangent operator at earlier times is applied to the state first. This $s$-dependent operator can induce a curve $c:[0,1] \mapsto \mathcal{H}$ such that

$$
c(s):=\hat{O}(s)|R\rangle, \quad c(0)=|R\rangle, \quad c(1)=|T\rangle .
$$

This curve is determined by the generator set $(E)$ and the coefficients $\left(Y_{I}\right)$ of tangent operator which are shown in eq. (4.18). Let us assume the image of the curve is $|\psi(s)\rangle$. We can compute the length of this curve by the Fubini-Study metric

$$
\mathcal{L}[c]:=\int_{0}^{1}\left[\left.|| \partial_{s}|\psi(s)\rangle\right|^{p}-\left|\left\langle\psi(s)\left|\partial_{s}\right| \psi(s)\right\rangle\right|^{p} \mid\right]^{1 / p} \mathrm{~d} s .
$$

This paper will focus on the $L^{1}$ normal, i.e., $p=1$ because it was shown that $p=1$ case leads that the complexity density resembles the divergence structure of holographic complexity [15].

The complexity between the states $|T\rangle$ and $|R\rangle$ is given by the following optimization problem,

$$
\begin{aligned}
\mathcal{C}(|T\rangle,|R\rangle):= & \min \{\mathcal{L}[c]|\forall c:[0,1] \mapsto \mathcal{H}, c(0)=| R\rangle, c(1)=|T\rangle, \\
& \text { and } \left.\exists\left\{Y^{I}\right\} \text { such that } \frac{\mathrm{d}}{\mathrm{d} s} c(s)=\sum_{I} Y_{I}(s) M^{I} c(s)\right\} .
\end{aligned}
$$

By this definition, the choice of generator set $E$ may affect the complexity between two states. So far the generator set $E$ is arbitrary and there may be many possible choices. Finding the complexity in a very general generator set seems to be a too mathematical and technical problem. However, in this subsection, we want to compute the complexity between $\left|\operatorname{TFD}\left(t_{L}, t_{R}\right)\right\rangle$ and $|0\rangle$ which are related by the operators $\hat{U}_{a}^{\dagger}\left(t_{L}, t_{R}\right)$. Because the TFD states can be generated by some generators which form a $\mathrm{su}(1,1)$ Lie algebra, as will be shown in (4.30), we choose, as a minimal nontrivial generator set,

$$
E_{L}=\bigcup_{\vec{k}}\left\{\hat{L}_{+}^{(\vec{k})}, \hat{L}_{-}^{(\vec{k})}, \hat{L}_{0}^{(\vec{k})}\right\}
$$


with

$$
\begin{aligned}
\hat{L}_{+}^{(\vec{k})} & :=\hat{a}^{R \dagger}(\vec{k}) \hat{a}^{L \dagger}(\vec{k}), \\
\hat{L}_{-}^{(\vec{k})} & :=\hat{a}^{R}(\vec{k}) \hat{a}^{L}(\vec{k}), \\
\hat{L}_{0}^{(\vec{k})} & :=\frac{1}{2}\left[\hat{a}^{R}(\vec{k}) \hat{a}^{R \dagger}(\vec{k})+\hat{a}^{L}(\vec{k}) \hat{a}^{L \dagger}(\vec{k})-1\right] .
\end{aligned}
$$

which satisfies the $s u(1,1)$ Lie-algebra

$$
\left[\hat{L}_{0}^{(\vec{k})}, \hat{L}_{ \pm}^{(\vec{k})}\right]= \pm \hat{L}_{ \pm}^{(\vec{k})}, \quad\left[\hat{L}_{-}^{(\vec{k})}, \hat{L}_{+}^{(\vec{k})}\right]=2 \hat{L}_{0}^{(\vec{k})}
$$

In general, the tangent operator $\hat{T}(s)$ has the form

$$
\hat{T}(s)=\int \mathrm{d}^{d-1} k\left[\alpha_{+}(s) \hat{L}_{+}^{(\vec{k})}+\alpha_{-}(s) \hat{L}_{-}^{(\vec{k})}+\alpha_{0}(s) \hat{L}_{0}^{(\vec{k})}\right],
$$

and we have

$$
\hat{O}(s)=\overleftarrow{P} \exp \left[\int_{0}^{s} \hat{T}(s) \mathrm{d} s\right]
$$

In order to compute the complexity between $\left|\operatorname{TFD}\left(t_{L}, t_{R}\right)\right\rangle$ and $|0\rangle$, we need an $s$-dependent operator $\hat{O}(s)$ satisfying

$$
\hat{O}(0)=I, \quad \hat{O}(1)|0\rangle=\left|\operatorname{TFD}\left(t_{L}, t_{R}\right)\right\rangle .
$$

Since, for different $s_{1}$ and $s_{2}$, the generators $\hat{T}\left(s_{1}\right)$ and $\hat{T}\left(s_{2}\right)$ do not commute, we cannot drop the time order operator $\overleftarrow{P}$ in (4.27). However, as the generator set (4.23) forms a complete Lie-algebra, there are three functions $b(\vec{k}, s), c(\vec{k}, s)$ and $d(\vec{k}, s)$ so that the operator $\hat{O}(s)$ can have a "normal decomposition" by using the decomposition formula of the $\mathrm{su}(1,1)$ Lie-algebra $[15,41]$

$$
\begin{aligned}
\hat{O}(s)= & \left.\exp \left[\int \mathrm{d}^{d-1} k b(\vec{k}, s) \hat{L}_{+}^{(\vec{k})}\right] \exp \left[\int \mathrm{d}^{d-1} k c(\vec{k}, s) \hat{L}_{0}^{(\vec{k})}\right]\right] \times \\
& \exp \left[\int \mathrm{d}^{d-1} k d(\vec{k}, s) \hat{L}_{-}^{(\vec{k})}\right] .
\end{aligned}
$$

The requirement $\hat{O}(0)=I$ shows that $b(\vec{k}, 0)=c(\vec{k}, 0)=d(\vec{k}, 0)=0$. One important point of the decomposed form (4.29) is that

$$
|\psi(s)\rangle=\hat{O}(s)|0\rangle=\mathcal{N}(s) \exp \left[\int \mathrm{d}^{d-1} k b(\vec{k}, s) \hat{L}_{+}^{(\vec{k})}\right]|0\rangle,
$$

where $\mathcal{N}(s)$ is a normalization constant factor. The constraint eq. (4.28) with eqs. (4.15) and (4.16) yield

$$
b_{+}(\vec{k}, 1)=e^{-\omega_{\vec{k}}\left[1 / 2 T+i\left(t_{R}+t_{L}\right)\right]}=\tanh r_{\vec{k}},
$$

where $r_{\vec{k}}$ is defined by eq. (4.17). Following ref. [15], we can find the complexity between $\left|\operatorname{TFD}\left(t_{L}, t_{R}\right)\right\rangle$ and $|0\rangle$

$$
\mathcal{C}\left(\left|\operatorname{TFD}\left(t_{L}, t_{R}\right)\right\rangle,|0\rangle\right)=\min \left\{\frac{\Sigma_{d-1}}{2} \int_{0}^{1} \mathrm{~d} s \int \mathrm{d}^{d-1} k \frac{\left|\partial_{s} b(\vec{k}, s)\right|}{1-|b(\vec{k}, s)|^{2}}\right\}
$$


with the constraint eq. (4.31). The solution for this optimization problem has been shown [15]:

$$
b(\vec{k}, s)=\tanh \left(r_{\vec{k}} s\right)=\tanh \left\{s \cdot \operatorname{arctanh} e^{-\omega_{\vec{k}}\left[1 / 2 T+i\left(t_{R}+t_{L}\right)\right]}\right\}
$$

and the complexity is given by,

$$
\Sigma_{d-1}^{-1} \mathcal{C}\left(\left|\operatorname{TFD}\left(t_{L}, t_{R}\right)\right\rangle,|0\rangle\right)=\int \mathrm{d}^{d-1} k\left|r_{\vec{k}}\right|=\int \mathrm{d}^{d-1} k\left|\operatorname{arctanh} e^{-\omega_{\vec{k}}\left[1 / 2 T+i\left(t_{R}+t_{L}\right)\right]}\right|
$$

For the full conformal symmetry case, we have $\omega_{\vec{k}}=k$, then eq. (4.34) becomes

$$
\begin{aligned}
\Sigma_{d-1}^{-1} \mathcal{C}\left(\left|\operatorname{TFD}\left(t_{L}, t_{R}\right)\right\rangle,|0\rangle\right) & =S_{d-2} \int_{0}^{\infty} \mathrm{d} k k^{d-2}\left|\operatorname{arctanh} e^{-k\left[1 / 2 T+i\left(t_{R}+t_{L}\right)\right]}\right| \\
& =2^{d-1} S_{d-2} T^{d-1} \Xi_{d}(\tilde{t})
\end{aligned}
$$

where $S_{d-2}$ is the area of $(d-2)$-dimensional sphere and $\tilde{t}:=2\left(t_{L}+t_{R}\right) T . \Xi_{d}(\tilde{t})$ is a function defined as $(x:=k / 2 T)$

$$
\Xi_{d}(\tilde{t}):=\int_{0}^{\infty} x^{d-2}\left|\operatorname{arctanh} e^{-(1+i \tilde{t}) x}\right| \mathrm{d} x
$$

which is finite only when $d \geq 2$. It is more convenient to write the result in terms of the total energy of the system. For the free scalar field with conformal symmetry, the total energy $E$ is expressed by

$$
\frac{E}{\hbar \Sigma_{d-1}}=\int \mathrm{d} k^{d-1} \omega_{\vec{k}} e^{-\omega_{\vec{k}} /(2 T)}=S_{d-2} 2^{d} T^{d} \int_{0}^{\infty} x^{d-1} e^{-x} \mathrm{~d} x=S_{d-2} 2^{d} \Gamma(d+1) T^{d},
$$

so we have

$$
\mathcal{C}\left(\left|\operatorname{TFD}\left(t_{L}, t_{R}\right)\right\rangle,|0\rangle\right)=\frac{\Xi_{d}(\tilde{t})}{2 \Gamma(d+1)} \frac{E}{\hbar T} .
$$

The growth rate of the complexity between $\left|\operatorname{TFD}\left(t_{L}, t_{R}\right)\right\rangle$ and $|0\rangle$ can be expressed as

$$
\frac{\mathrm{d}}{\mathrm{d}\left(t_{L}+t_{R}\right)} \mathcal{C}\left(\left|\operatorname{TFD}\left(t_{L}, t_{R}\right)\right\rangle,|0\rangle\right)=\frac{E}{\hbar \Gamma(d+1)} \dot{\Xi}_{d}(\tilde{t})
$$

For small time $\tilde{t}$ we have the following expansion

$$
\Xi_{d}(\tilde{t})=\mathfrak{I}_{d}^{(0)}-\frac{1}{2} \mathfrak{I}_{d}^{(1)} \tilde{t}^{2}+\mathcal{O}\left(\tilde{t}^{4}\right)
$$

where $\mathfrak{I}_{d}^{(0)}=\Gamma(d)\left(2^{d}-1\right) \zeta(d) /\left[2^{d}(d-1)\right]$ and $\mathfrak{I}_{d}^{(1)}>0$. It is not easy to write down the analytic formula for $\mathfrak{I}_{d}(1)$ so the numerical computations shows that

$$
\mathfrak{I}_{3}^{(1)} \approx 0.07565, \quad \mathfrak{I}_{4}^{(1)} \approx 0.1639 .
$$

For large $\tilde{t}$ limit, i.e., in the late time limit, we can see that the phase factor $i k \tilde{t}$ makes a rapidly oscillation so the complexity becomes constant

$$
\lim _{t \rightarrow \infty} \Xi_{d}(\tilde{t})=\mathfrak{I}_{d}^{(0)} \vartheta,
$$



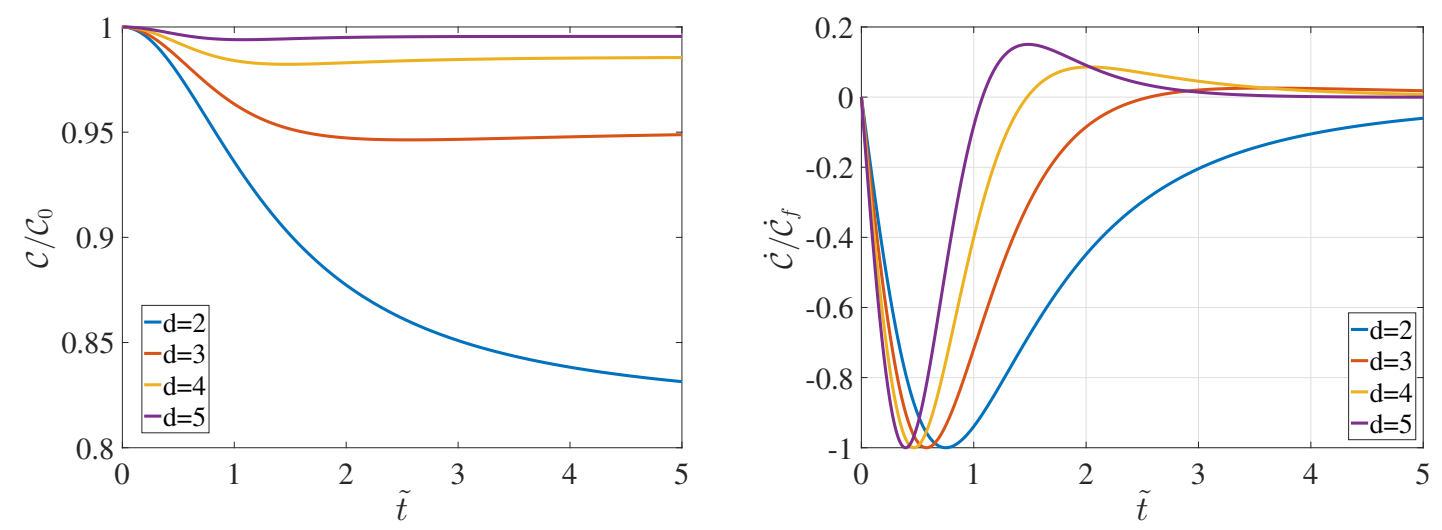

Figure 7. The numerical values of $\mathcal{C}$ and $\dot{\mathcal{C}}$ at $d=2,3,4,5$. Here $\mathcal{C}_{0}$ is the complexity when $\tilde{t}=0$ and $\dot{\mathcal{C}}_{f}=-\dot{\mathcal{C}}_{\text {min }}$. They show that $\dot{\mathcal{C}}$ will first decease linearly with respective to time $\tilde{t}$ and then increase later. Finally, the $\dot{\mathcal{C}}$ goes to zero.

with a positive constant $\vartheta \approx 0.986$. Thus we conclude

$$
\frac{\mathrm{d}}{\mathrm{d}\left(t_{L}+t_{R}\right)} \mathcal{C}\left(\left|\operatorname{TFD}\left(t_{L}, t_{R}\right)\right\rangle,|0\rangle\right)=\left\{\begin{array}{cl}
-\frac{E}{\hbar \Gamma(d+1)} \Im_{d}^{(1)} \tilde{t} & \text { for }|\tilde{t}| \ll 1, \\
0 & \text { for }|\tilde{t}| \rightarrow \infty .
\end{array}\right.
$$

In figure 7 , the values of $\mathcal{C}\left(\mid \operatorname{TFD}\left(t_{L}, t_{R}\right)\right.$ and its growth rates for different $\tilde{t}$ are shown. We see that in the Fubini-Study metric method, the complexity growth rate between a TFD state and its corresponding vacuum state is negative for small time and increases later, finally goes to zero in the large time limit. This is very different from the CV and CA conjectures and also the FS method, which will be considered in the following subsection.

\subsection{Finsler geometry (FG)}

In this subsection, we will use another field theoretic method proposed by ref. [16] to compute the time dependence of the complexity in the TFD state. Ref. [16] first try to define the complexity for an operator and then define the complexity between two states. Let us first make a brief review on this method.

For a given generator set $E=\left\{M^{1}, M^{2}, \cdots\right\}$, all the operators generated by (eqs. (4.19) and (4.18))

$$
\hat{O}(s):=\overleftarrow{P} \exp \left[\int_{0}^{s} \hat{T}(\tilde{s}) \mathrm{d} \tilde{s}\right], \quad \hat{T}(s)=\sum_{I} Y_{I}(s) M^{I}
$$

form an operator set $\mathcal{O}$ where the identity operator $I$ is also included. Eq. (4.44) defines a curve, $c(s)$, in $\mathcal{O}, c:[0,1] \mapsto \mathcal{U}$. The length of the curve may be defined as

$$
L[c]:=\int_{0}^{1} \mathrm{~d} s F[c(s), \hat{T}(s)],
$$

where the Finsler structure $F[c(s), \hat{T}(s)]$ is always positive and some functional of $Y_{I}(s)$, which depends on the choice of the generators $\hat{T}(s)$ of the curve $c(s)$. The explicit form of the Finsler structure will be explained later on. 
Once the length is defined, the complexity of any operator $\hat{O}$ belonging to $\mathcal{O}$ is given by the minimal length from the identity:

$$
\mathcal{C}(\hat{O}):=\min \{L[c] \mid \forall c:[0,1] \mapsto \mathcal{O} \text {, s.t., } c(0)=I \text { and } \exists \lambda \neq 0, c(1)=\lambda \hat{O}\} .
$$

After defining the complexity of an operator, we may define the complexity from one state to another state as

$$
\mathcal{C}\left(\left|\psi_{2}\right\rangle,\left|\psi_{1}\right\rangle\right):=\min \left\{\mathcal{C}(\hat{O}) \mid \forall \hat{O} \in \mathcal{O}, \text { s.t., } \hat{O}\left|\psi_{1}\right\rangle \sim\left|\psi_{2}\right\rangle\right\}
$$

where the notation $\sim$ means that two state can be different by a nonzero complex constant. Thus, there are three steps to find the complexity between two states. Firstly, we have to find the complexity of all operators in $\mathcal{O}$. Then, we have to find all the operators which can change the reference state to the target state. Finally, we need to find the minimal complexity of these operators. For some cases where we only care about the complexity between states it is not necessary to compute the complexity of all the operators. Instead, we can directly solve the following optimization problem

$\mathcal{C}\left(\left|\psi_{2}\right\rangle,\left|\psi_{1}\right\rangle\right):=\min \left\{L[c] \mid \forall c:[0,1] \rightarrow \mathcal{O}\right.$, s.t., $\hat{O}(s)=c(s), \hat{O}(0)=I$ and $\left.\hat{O}(1)\left|\psi_{1}\right\rangle \sim\left|\psi_{2}\right\rangle\right\}$.

In some cases, this optimization problem is easier to handle than finding complexity of operators.

In ref. [16], a very general generator set formed by creation and annihilation operators is considered. Although this makes the generator set big enough, it makes the optimization problems (4.46) and (4.47) hard to solve exactly. We want to choose a smaller generator set for the TFD states so that we can compute the complexity analytically. (If we choose a larger generator set, the complexity tends to decrease because there may be a "shorter path" in the enlarged generator space.) Also, in order to make a comparison with the results in the Fubini-Study metric, we will use a generator set, which is defined by (4.23). In this case, the operator set $\mathcal{O}$ is just the infinite direct product of $\mathrm{SU}(1,1)$ group. Any operator in $\mathcal{O}$ can be parameterized uniquely by three complex-valued functions $\gamma_{+}(\vec{k}), \gamma_{-}(\vec{k})$ and $\gamma_{0}(\vec{k})$.

$$
\begin{aligned}
\hat{U}\left[\gamma_{+}(\vec{k}), \gamma_{-}(\vec{k}), \gamma_{0}(\vec{k})\right]= & \left.\exp \left[\int \mathrm{d}^{d-1} k \gamma_{+}(\vec{k}) \hat{L}_{+}^{(\vec{k})}\right] \exp \left[\int \mathrm{d}^{d-1} k \ln \gamma_{0}(\vec{k}) \hat{L}_{0}^{(\vec{k})}\right]\right] \times \\
& \exp \left[\int \mathrm{d}^{d-1} k \gamma_{-}(\vec{k}) \hat{L}_{-}^{(\vec{k})}\right] .
\end{aligned}
$$

We find from (4.16) that $\left|\operatorname{TFD}\left(t_{L}, t_{R}\right)\right\rangle \sim \hat{U}\left[\gamma_{+}(\vec{k}), \gamma_{-}(\vec{k}), \gamma_{0}(\vec{k})\right]|0\rangle$ if and only if $\gamma_{+}=$ $e^{-\omega_{\vec{k}}\left[1 / 2 T+i\left(t_{R}+t_{L}\right)\right]}$. Let us take

$$
\hat{U}_{T}\left(t_{L}, t_{R}\right):=\exp \left[\int \mathrm{d}^{d-1} k e^{-\omega_{\vec{k}}\left[1 / 2 T+i\left(t_{R}+t_{L}\right)\right]} \hat{L}_{+}^{(\vec{k})}\right] .
$$

Thus the set of all the operators which can change from $|0\rangle$ to $\left|\operatorname{TFD}\left(t_{L}, t_{R}\right)\right\rangle$ is

$$
\mathcal{D}:=\left\{\hat{U}\left[\gamma_{+}(\vec{k}), \gamma_{-}(\vec{k}), \gamma_{0}(\vec{k})\right] \mid \forall \gamma_{-}, \gamma_{0} \in \mathbb{C}, \gamma_{+}=e^{-\omega_{\vec{k}}\left[1 / 2 T+i\left(t_{R}+t_{L}\right)\right]}\right\} .
$$


The complexity between $|0\rangle$ to $\left|\operatorname{TFD}\left(t_{L}, t_{R}\right)\right\rangle$ is given by

$$
\mathcal{C}\left(\left|\operatorname{TFD}\left(t_{L}, t_{R}\right)\right\rangle,|0\rangle\right)=\min \{\mathcal{C}(\hat{U}) \mid \forall \hat{U} \in \mathcal{D}\}
$$

In order to proceed, we need to obtain the explicit form of the Finsler structure in the generator set $E_{L}$. Relegating technical details to appendix $\mathrm{C}$ we here present a final result. For any generator $\hat{T}(s)$ expanded in the basis $E_{L}$

$$
\hat{T}(s)=\int \mathrm{d}^{d-1} k\left[\alpha_{+}(s, \vec{k}) \hat{L}_{+}^{(\vec{k})}+\alpha_{0}(s, \vec{k}) \hat{L}_{0}^{(\vec{k})}+\alpha_{-}(s, \vec{k}) \hat{L}_{-}^{(\vec{k})}\right] .
$$

the Finsler structure is given by

$$
\left.F\right|_{E_{L}}=\ell_{0} \Sigma_{d-1} \int \mathrm{d}^{d-1} k\left[\left\|\alpha_{+}(s, \vec{k})\right\|+\left\|\alpha_{-}(s, \vec{k})\right\|+\left\|\alpha_{0}(s, \vec{k})\right\|\right],
$$

where $\ell_{0}$ is a free parameter to be chosen later. Here we chose the $L_{1}$ norm, which is a natural generalization of the gate complexity to a continuous system. Suppose $\hat{O}(4.44)$ is constructed by the sequence of the discrete fundamental operators (logic gates) which are generated by $\hat{\sigma}_{1}, \hat{\sigma}_{2}, \cdots$ at the time $t_{1}, t_{2}, \cdots$. We can express a generator in terms of $\delta$-functions

$$
\hat{T}(t)=\delta\left(t-t_{1}\right) \hat{\sigma}_{1}+\delta\left(t-t_{2}\right) \hat{\sigma}_{2}+\cdots=\sum_{n=0} \delta\left(t-t_{n}\right) \hat{\sigma}_{n} .
$$

$L_{1}$ norm yields the total number of fundamental operators, so the curve of minimal length just corresponds to the design of minimal required gates. In this sense, $L_{1}$ is a natural generalization of the gate complexity to a continuous system. For more technical argument we refer to section $\mathrm{E}$ of ref. [20]

Based on the detailed computation in appendix D, it turns out that

$$
\mathcal{C}\left(\left|\operatorname{TFD}\left(t_{L}, t_{R}\right)\right\rangle,|0\rangle\right)=\ell_{0} \Sigma_{d-1} \int \mathrm{d}^{d-1} k\left\|\gamma_{+}(\vec{k})\right\|
$$

Therefore, we have

$$
\mathcal{C}\left(\left|\operatorname{TFD}\left(t_{L}, t_{R}\right)\right\rangle,|0\rangle\right)=\ell_{0} \Sigma_{d-1} \int_{0}^{\infty} k^{d-2}\left\|e^{-\omega_{\vec{k}}\left[1 / 2 T+i\left(t_{R}+t_{L}\right)\right]}\right\| \mathrm{d} k .
$$

Using the definition $\|\cdot\|$ in eq. (C.9), we finally obtain the complexity between $|0\rangle$ to $\left|\operatorname{TFD}\left(t_{L}, t_{R}\right)\right\rangle$ :

$$
\mathcal{C}\left(\left|\operatorname{TFD}\left(t_{L}, t_{R}\right)\right\rangle,|0\rangle\right)=\ell_{0} \Sigma_{d-1} S_{d-2} 2^{d-1} T^{d-1} \Omega_{d}(\tilde{t})=\ell_{0} \frac{\Omega_{d}(\tilde{t})}{2 \Gamma(d+1)} \frac{E}{\hbar T},
$$

where $S_{d-2}$ is the area of $(d-2)$-dimensional sphere, $\tilde{t}:=2\left(t_{L}+t_{R}\right) T$,

$$
\Omega_{d}(\tilde{t}):=\int_{0}^{\infty} x^{d-2} e^{-x}(|\cos x \tilde{t}|+|x \tilde{t}| \cdot|\sin x \tilde{t}|) \mathrm{d} x,
$$

and the total energy $E$ is given by eq. (4.37). 

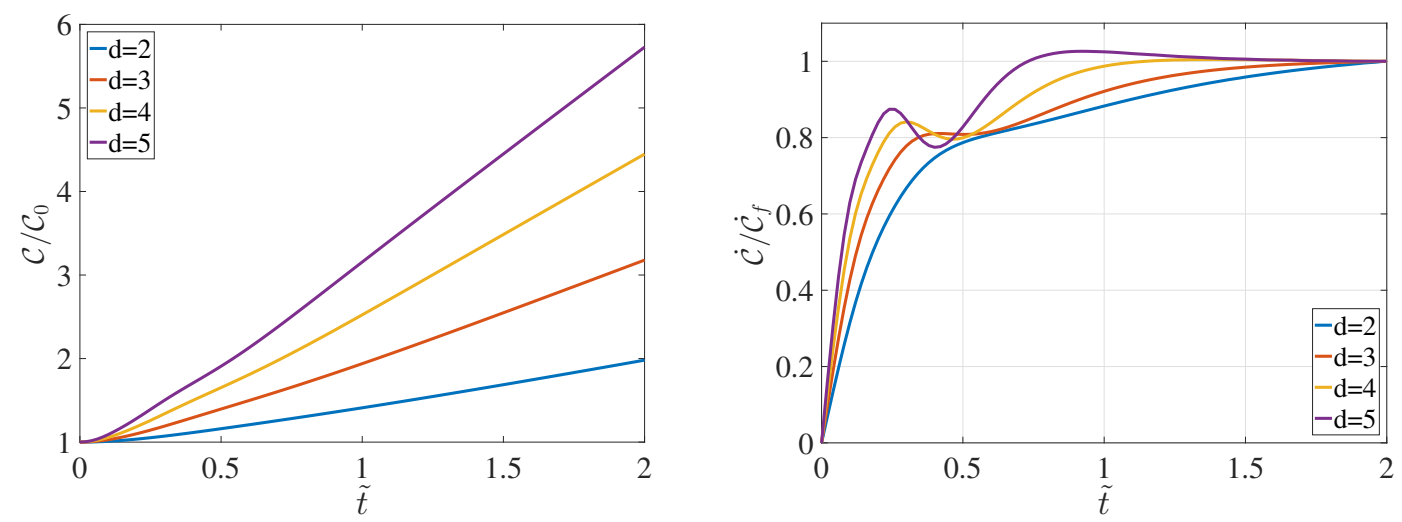

Figure 8. The numerical values of complexity and its growth rate at $d=2,3,4,5 . \mathcal{C}_{0}$ is the complexity at $\tilde{t}=0$ and $\dot{\mathcal{C}}_{f}$ is the growth rate at the late time limit. From the left panel, we see that $\mathcal{C}$ will monotonously increase with respective to time $\tilde{t}$. For small $\tilde{t}, \dot{\mathcal{C}}$ will linearly depends on $\tilde{t}$. For large $\tilde{t}, \dot{\mathcal{C}}$ will tend to increase linearly with respective to $\tilde{t}$ and $\dot{\mathcal{C}}$ tends to a constant. For large $d$, we obtain similar behaviours.

For small $\tilde{t}$

$$
\Omega_{d}(\tilde{t})=\Gamma(d-1)+\frac{\Gamma(d+1)}{2} \widetilde{t}^{2}+\mathcal{O}\left(\tilde{t}^{4}\right)
$$

and for large $\tilde{t}$

$$
\Omega_{d}(\tilde{t})=\frac{2}{\pi}[\Gamma(d-1)+\Gamma(d) \tilde{t}][1+\mathcal{O}(1 / \tilde{t})]
$$

Thus we see that

$$
\frac{\mathrm{d}}{\mathrm{d}\left(t_{L}+t_{R}\right)} \mathcal{C}\left(\left|\operatorname{TFD}\left(t_{L}, t_{R}\right)\right\rangle,|0\rangle\right)= \begin{cases}\ell_{0} E \tilde{t} / \hbar & \text { for }|\tilde{t}| \ll 1 \\ \ell_{0} \frac{E}{\hbar d} & \text { for }|\tilde{t}| \gg 1\end{cases}
$$

For the intermediate time, we can compute $\Omega_{d}(\tilde{t})$ analytically but it is not so illuminating. Therefore, we show a numerical plot for $\Omega_{4}(\tilde{t})$ and $\dot{\Omega}_{4}(\tilde{t})$ in the figure 8. For lager $d>4$, the behavior is similar.

Note that the linear $\tilde{t}$ dependence of the complexity in the late time limit comes from $|x \tilde{t}|$ in eq. (4.59), which is due to our definition of $\|\cdot\|$ in eq. (C.9). If we use the definition $\|\cdot\|$ in eq. (C.8) then the complexity will be constant independent of time, which is the same as the FS case. Therefore, our result here is not so robust. It should be understood as one example to define the field theory complexity showing the linear-time complexity.

The complexity growth rate is positive and linearly dependent of $t$ at the early time $\left(\left(t_{L}+t_{R}\right) T \ll 1\right)$, which is the same as the prediction of the CV conjecture. In the late time limit $\left(\left(t_{L}+t_{R}\right) T \gg 1\right)$, eq. (4.62) is constant and proportional to $T^{d}$. In the planar symmetry asymptotic AdS black hole, the total ADM mass $M$ is also proportional to $T^{d}$. Thus we see that the complexity growth rate is similar to the predictions of both the CV and CA conjectures in the late time limit. The free parameter $\ell_{0}$ in eq. (4.57) can be determined if we require that the complexity growth rate saturate to the Lloyd's bound at 
$t_{L}+t_{R} \rightarrow \infty$. We see that if we take

$$
\ell_{0}=\frac{2 d}{\pi}
$$

the complexity growth rate at the late time limit saturates to the Lloyd's bound. However, it turned out that the subleading term in eq. (4.61) is positive so the complexity growth rate will approach to the limiting value from the larger value. Thus, like the CA conjecture, at large time region, the complexity growth rate violate the Lloyd's bound with this choice of $\ell_{0}$.

\section{Summary}

In this paper, we have computed the complexity of the time dependent TFD states and their growth rates by four different methods, two holographic and two field theory methods. Two holographic methods are based on the "complexity-action" (CA) conjecture or "complexity-volume" (CV) conjecture. Two quantum field theoretic methods are based on the Fubini-Study metric (FS) or the Finsler geometry (FG). In particular, for holographic computation, we have proposed a modified $\mathrm{CA}$ and $\mathrm{CV}$ conjectures between two TFD states, $\left|\mathrm{TFD}_{2}\right\rangle$ and $\left|\mathrm{TFD}_{1}\right\rangle(2.1)$

$$
\begin{aligned}
& \mathcal{C}_{V}\left(\left|\mathrm{TFD}_{2}\right\rangle,\left|\mathrm{TFD}_{1}\right\rangle\right) \equiv\left|\mathcal{C}_{V}^{(1)}-\mathcal{C}_{V}^{(2)}\right| \\
& \mathcal{C}_{A}\left(\left|\mathrm{TFD}_{2}\right\rangle,\left|\mathrm{TFD}_{1}\right\rangle\right) \equiv\left|\mathcal{C}_{A}^{(1)}-\mathcal{C}_{A}^{(2)}\right|
\end{aligned}
$$

where $\mathcal{C}_{V}^{(i)}$ and $\mathcal{C}_{A}^{(i)}$ are the original $\mathrm{CV}$ and $\mathrm{CA}$ conjectures for the $\left|\mathrm{TFD}_{i}\right\rangle$ state. It is similar to the 'complexity of formation' proposed in [29] but there is a subtle difference in that here we do not assume any reference state [16]. These modified versions yield finite values agreeing to the field theory computation for a static case [16]. For a concrete example in this paper we consider the complexity between the time-dependent TFD state and its corresponding vacuum state. We call it 'complexity' for simplicity. In the table 1 , we list some main differences between the original and modified CV and CA conjectures.

Our main results for the time dependent complexity for the TFD states are summarized in table 2. As a companion to table 2, for readers' convenience, we show a schematic plot, figure 9 of which precise information can be found in figures $2,3,5,7$, and 8 . We define a common time $\bar{t}=t_{L}+t_{R}$ for all cases.

If $\bar{t}=0$, four different methods give similar results but give different predictions on the time evolution of the complexity. At early time, both the CV conjecture and FG method predict that the complexity will increase as $\bar{t}^{2}$ while the FS method predicts that the complexity will decrease as $-\vec{t}^{2}$. The CA conjecture says that for $d>2$ the complexity does not change until a critical time and after then it will decrease. For $d=2$, it decreases first and increases as time goes on.

In the late time limit, the CA conjecture, CV conjecture and FG method predict that the complexity will increase linearly in $\bar{t}$ and the growth rate will be proportional to the total energy of the system. On the contrary, the FS method shows that the complexity 


\begin{tabular}{|c|c|c|}
\hline & Original conjectures & Modified versions \\
\hline Non-negativeness & CV: Yes, CA: No & Yes \\
\hline Reference state & $\begin{array}{c}\text { A particular but unknown } \\
\text { reference state }\left(\left|R^{*}\right\rangle\right) \text { is assumed }\end{array}$ & No need \\
\hline Is complexity finite? & Always infinite & $\begin{array}{c}\text { Finite between } \\
\text { two TFD states }\end{array}$ \\
\hline $\begin{array}{c}\text { Can compute complexity } \\
\text { between two TFD states? }\end{array}$ & $\begin{array}{c}\text { Can be computed } \\
\text { with a given particular } \\
\text { reference state }\left|R^{*}\right\rangle\end{array}$ & $\begin{array}{c}\text { Can be computed } \\
\text { with an arbitrary } \\
\text { reference state } \phi\end{array}$ \\
\hline Complexity of formation & \begin{tabular}{c} 
only \\
\hline
\end{tabular}
\end{tabular}

Table 1. The differences between the original CV and CA conjectures and our modified versions. The complexity of formation is defined in ref. [29]. For a given reference state $|\phi\rangle$, the complexity of formation of state $\left|\psi_{2}\right\rangle$ from $\left|\psi_{1}\right\rangle$ is defined by $\Delta_{\phi} \mathcal{C}\left(\left|\psi_{2}\right\rangle,\left|\psi_{1}\right\rangle\right):=\mathcal{C}\left(\left|\psi_{2}\right\rangle,|\phi\rangle\right)-\mathcal{C}\left(\left|\psi_{1}\right\rangle,|\phi\rangle\right)$.

\begin{tabular}{|c|c|c|c|c|}
\hline & $\mathrm{CA}$ & $\mathrm{CV}$ & $\mathrm{FS}$ & $\mathrm{FG}$ \\
\hline$t_{L}+t_{R}=0$ & $\mathcal{C} \propto \frac{E}{\hbar T}$ & $\mathcal{C} \propto \frac{E}{\hbar T}$ & $\mathcal{C} \propto \frac{E}{\hbar T}$ & $\mathcal{C} \propto \frac{E}{\hbar T}$ \\
\hline early time & $\begin{array}{c}\dot{\mathcal{C}}=0 \text { if } d>2 \\
\dot{\mathcal{C}}=-\infty \text { if } d=2\end{array}$ & $\dot{\mathcal{C}} \propto \bar{t}$ & $\dot{\mathcal{C}} \propto-\bar{t}$ & $\dot{\mathcal{C}} \propto \bar{t}$ \\
\hline late time & $\dot{\mathcal{C}}=\frac{2 E}{\pi \hbar}$ & $\dot{\mathcal{C}}=\frac{2 E}{\pi \hbar}$ & $\dot{\mathcal{C}}=0$ & $\dot{\mathcal{C}}=\frac{2 E}{\pi \hbar}$ \\
\hline $\operatorname{sign}(\dot{\mathcal{C}})$ & indefinite & + & indefinite & + \\
\hline Lloyd's bound & broken & satisfied & saisfied & broken \\
\hline
\end{tabular}

Table 2. The summary of the complexity between the time-dependent TFD state and its corresponding vacuum state in four different methods. $\bar{t}=t_{L}+t_{R}$ and $E$ is the total energy of the system. We have set $\ell / \ell_{\mathrm{AdS}}=4 \pi^{2} \hbar /(d-1)$ for the $\mathrm{CV}$ conjecture, $\ell_{0}=2 d / \pi$ for the FG method, and the speed of light $c=1$.

in the late time limit will keep constant rather than increasing. The CV conjecture and FG method show that the complexity will monotonically increase for $\bar{t}>0$ while the CA conjecture and FS method show that the complexity first decreases and then increases. The Lloyd's bound is satisfied only for the CV conjecture and FS method. The Lloyd's bound is also satisfied for the CA conjecture and FG method in the late time limit, but it is weakly violated in the intermediate time. We have set $\ell / \ell_{\mathrm{AdS}}=4 \pi^{2} \hbar /(d-1)$ for the $\mathrm{CV}$ conjecture, $\ell_{0}=2 d / \pi$ for the FG method. With other choices, the growth rate saturate to some value which is not the Lloyd's bound.

The results summarized in table 2 seem to give us some pieces of information to judge which are appropriate methods to compute the complexity among two holographic conjectures and two quantum field theory proposals. For examples, if we expect that the complexity should increase with time then it seems that the CV conjecture and FG method are favored. The similarity between the CV conjecture and the FG method in the early and 

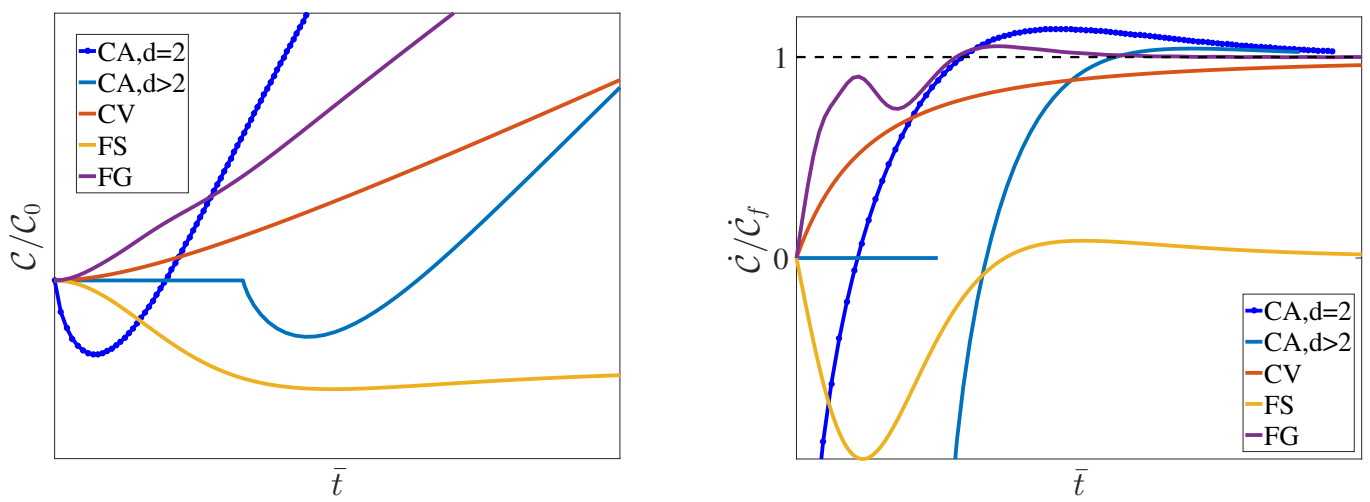

Figure 9. Schematic plots for the complexity by four methods: the holographic CV and CA conjecture and the field theoretic FS and FG methods. It is obtained from figures $2,3,5,7$, and 8 . $\bar{t}=t_{L}+t_{R}$. Left: $\mathcal{C} / \mathcal{C}_{0}$, where $\mathcal{C}_{0}$ is the complexity at $\bar{t}=0$. Right: $\dot{\mathcal{C}} / \dot{\mathcal{C}}_{f}$, where $\dot{\mathcal{C}}_{f}$ is the growth rate at $\bar{t} \rightarrow \infty$ in $\mathrm{CA}, \mathrm{CV}$ and FG methods, and $\dot{\mathcal{C}}_{f}=-\dot{\mathcal{C}}_{\text {min }}$ in the FS method.

late time limit seems to show these two proposal are more correlated while they are different in the intermediate time regime. Note that the FS method is quite different from all the other methods. In particular, the FS method shows the complexity will keep constant in the late time limit. Because the linear growth of the complexity in the late time limit has much evidence both in quantum information theory and holography $[6,7,9,13,42-44]$ it seems to be a challenge to the FS method.

However, there is also a caveat in the FG method. The results of the FG method depend on the definition of the Finsler structure. Our result here should be understood as just one example to define the Finsler structure displaying the linear-time complexity in the late time limit and showing similar behaviors to holographic complexity. For both the FS and FG method, the complexity also depend on the generator set. We have chosen a small generator set to make the TFD states so that we can compute the complexity analytically. If we choose another generator set the complexity may or may not change. Therefore, it will be interesting to investigate how much our results are robust under different choices of generator sets and/or different choices of the Finsler structures.

\section{Acknowledgments}

The work of K.-Y. Kim and C. Niu was supported by Basic Science Research Program through the National Research Foundation of Korea(NRF) funded by the Ministry of Science, ICT \& Future Planning(NRF- 2017R1A2B4004810) and GIST Research Institute(GRI) grant funded by the GIST in 2017. C.Y. Zhang is supported by National Postdoctoral Program for Innovative Talents BX201600005. We also would like to thank the APCTP(Asia-Pacific Center for Theoretical Physics) focus program, "Geometry and Holography for Quantum Criticality" in Pohang, Korea for the hospitality during our visit, where part of this work was done. 


\section{A Bogoliubov transformation and TFD states}

To construct a TFD state, we may use a bogoliubov transformation from the vacuum state defined by any chosen annihilation operators [16]. Let us first decompose the Hilbert space $\mathcal{H}=\mathcal{H}_{L} \times \mathcal{H}_{R}$ and define two groups of annihilation and creation operators $\left\{\hat{a}_{\vec{k}_{i}}^{L}, \hat{a}_{\vec{k}_{i}}^{R}\right\}$ and $\left\{\hat{a}_{\vec{k}_{i}}^{L \dagger}, \hat{a}_{\vec{k}_{i}}^{R \dagger}\right\}$, which define a vacuum state $|0\rangle$ :

$$
\hat{a}_{\vec{k}_{i}}^{L}|0\rangle=\hat{a}_{\vec{k}_{i}}^{R}|0\rangle=0, \quad \forall \vec{k}_{i} .
$$

However, the decomposition $\mathcal{H}=\mathcal{H}_{L} \times \mathcal{H}_{R}$ is not unique. One can choose another decomposition such that $\mathcal{H}=\mathcal{H}_{D} \times \mathcal{H}_{U}$ with the annihilation and creation operators $\left\{\hat{b}_{\vec{k}_{i}}^{D}, \hat{b}_{\vec{k}_{i}}^{U}\right\}$ and $\left\{\hat{b}_{\vec{k}_{i}}^{D \dagger}, \hat{b}_{\vec{k}_{i}}^{U \dagger}\right\}$. The annihilation operators $\left\{\hat{b}_{\vec{k}_{i}}^{D}, \hat{b}_{\vec{k}_{i}}^{U}\right\}$ also define a vacuum state $|B\rangle$ such that $\hat{b}_{\vec{k}_{i}}^{U}|B\rangle=\hat{b}_{\vec{k}_{i}}^{D}|B\rangle=0$ for $\forall \vec{k}_{i}$. In general, two kinds of decompositions can have no special relationship. However, if we demand that they satisfy the following relationship

$$
\left[\begin{array}{c}
\hat{b}_{\vec{k}}^{D} \\
\hat{b}_{\vec{k}}^{+}
\end{array}\right]=c_{\vec{k}}\left[\begin{array}{cc}
1 & -e^{-\pi \omega_{\vec{k}} / a} \\
-e^{-\pi \omega_{\vec{k}} / a} & 1
\end{array}\right]\left[\begin{array}{c}
\hat{a}_{\overrightarrow{\vec{k}}}^{L} \\
\hat{a}_{\vec{k}}^{R \dagger}
\end{array}\right]
$$

for a normalization factor $c_{\vec{k}}$ which makes the operators $\left\{\hat{b}_{\vec{k}_{i}}^{D}, \hat{b}_{\vec{k}_{i}}^{U}\right\}$ and $\left\{\hat{b}_{\vec{k}_{i}}^{D \dagger}, \hat{b}_{\vec{k}_{i}}^{U \dagger}\right\}$ to satisfy the canonical commutation relation, ref. [16] and ref. [45] have proven that the vacuum state $|B\rangle$ can be expressed by

$$
|B\rangle \propto \prod_{\vec{k}_{i}} \sum_{n=0}^{\infty} e^{-\pi n \omega_{\vec{k}_{i}} / a}\left|n, \vec{k}_{i}\right\rangle_{L}\left|n, \vec{k}_{i}\right\rangle_{R}
$$

There is a non-unitary operator

$$
\hat{U}_{a}^{\dagger}:=\prod_{\vec{k}_{i}} \exp \left(e^{-\pi \omega_{\vec{k}_{i}} / a} \hat{a}_{\vec{k}_{i}}^{R \dagger} \hat{a}_{\vec{k}_{i}}^{L \dagger}\right)=\exp \left[\int \mathrm{d}^{d-1} k e^{-\pi \omega_{\vec{k}} / a} \hat{a}^{R \dagger}(\vec{k}) \hat{a}^{L \dagger}(\vec{k})\right],
$$

which can convert the vacuum state $|0\rangle$ into the $|B\rangle$, i.e., $|B\rangle \propto \hat{U}_{a}^{\dagger}|0\rangle$. In the second equality, the discrete form has been converted into a continuous form.

In order to prove the state $|B\rangle$ is a TFD state, the easiest way is to find the reduced density matrix in the projected Hilbert space $\mathcal{H}_{L}$ or $\mathcal{H}_{R}$. Ref. [45] has shown that the state in eq. (A.3) has the following reduced density matrix

$$
\hat{\rho}_{L}=\hat{\rho}_{R}=\frac{1}{Z} \prod_{\vec{k}_{i}} \sum_{n=0}^{\infty} \exp \left(-2 \pi n \omega_{\vec{k}_{i}} / a\right)\left|n, \vec{k}_{i}\right\rangle\left\langle n, \vec{k}_{i}\right|,
$$

where the factor $1 / Z$ insures that $\operatorname{Tr} \hat{\rho}_{L}=\operatorname{Tr} \hat{\rho}_{R}=1$. We see that this is the density matrix for the system of free bosons with temperature $T=a / 2 \pi$. Thus, the projected states of $|B\rangle$ in Hilbert space $\mathcal{H}_{L}$ and $\mathcal{H}_{R}$ are two thermofield state. This shows that $|B\rangle$ is a TFD state with temperature $T=a / 2 \pi$. 


\section{B Fubini-Study metric}

Let us consider an $n$-dimensional Hilbert space $\mathcal{H}$. Any two vector $\left|\psi_{1}\right\rangle$ and $\left|\psi_{2}\right\rangle$ describe the same state if there is a nonzero complex number $c$ such that $\left|\psi_{1}\right\rangle=c\left|\psi_{2}\right\rangle$. This means that the different states of the Hilbert space $\mathcal{H}$ form a complex projective space $\mathbb{C P}^{n}$. As $\mathbb{C P}^{n}=S^{2 n+1} / S^{1}$, we can use the length of geodesic curve in $S^{2 n+1} / S^{1}$ to build the distance of two states. It turns out that this distance is the Fubini-Study distance, which is

$$
D_{F S}(|\psi\rangle,|\phi\rangle):=\arccos (|\langle\psi \mid \phi\rangle|) \in[0, \pi / 2] .
$$

In this equation, the state vectors should satisfy $\langle\psi \mid \psi\rangle=\langle\phi \mid \phi\rangle=1$. This expression can be generalized to the infinite dimensional cases. To obtain the line element in a local form, let us assume

$$
|\phi\rangle=\mathcal{N}(|\psi\rangle+\mathrm{d}|\psi\rangle)
$$

and expand to second order in the vector $\mathrm{d}|\psi\rangle . \mathcal{N}$ is the normalization factor for $\langle\phi \mid \phi\rangle=1$. The result is the Fubini-Study metric

$$
\mathrm{d} s_{F S}^{2}(|\psi\rangle)=\langle\mathrm{d} \psi \mid \mathrm{d} \psi\rangle^{2}-|\langle\mathrm{d} \psi \mid \psi\rangle|^{2} .
$$

For any curve $l:\left[s_{i}, s_{f}\right] \mapsto \mathcal{H}$ such that $l(t)=|\psi(t)\rangle$, its length is defined by

$$
L^{2}[l]:=\int_{s_{i}}^{s_{f}} \mathrm{~d} s_{F S}(|\psi(t)\rangle)=\int_{s_{i}}^{s_{f}} \mathrm{~d} t \sqrt{\left.\left|\partial_{t}\right| \psi(t)\right\rangle\left.\right|^{2}-\left|\left\langle\psi(t)\left|\partial_{t}\right| \psi(t)\right\rangle\right|^{2}}
$$

If there is no restriction for the curve, the length of the geodesic connecting any two states is given by eq. (B.1). We can define the complexity for two states

$$
\mathcal{C}(|\psi\rangle,|\phi\rangle):=D_{F S}(|\psi\rangle,|\phi\rangle)=\arccos (|\langle\psi \mid \phi\rangle|) \in[0, \pi / 2] .
$$

However, in the case that the curve can only be generated by some appointed generator set $E$, the minimal length of the curves may be different from the eq. (B.1) and we have to solve the following optimization problem

$$
\left.\mathcal{C}(|\psi\rangle,|\phi\rangle, E):=\min _{E}\left\{L^{2}[l]\left|l\left(s_{i}\right)=\right| \psi\right\rangle, l\left(s_{f}\right)=|\phi\rangle\right\} .
$$

As noted by ref. [15], it is not necessary to restrict the line element in $L^{2}$ normal. Then for more general case, we can define the general Fubini-Study metric by $L^{p}$ normal, which reads

$$
\mathrm{d} s:=\left[\left.|| \partial_{s}|\psi(s)\rangle\right|^{p}-\left|\left\langle\psi(s)\left|\partial_{s}\right| \psi(s)\right\rangle\right|^{p} \mid\right]^{1 / p} .
$$

\section{Finsler structure in the generator set $E_{L}$}

In this appendix, we explain how to obtain the explicit functional form of the Finsler structure. We start with the proposal for a more general case in ref. [16] and we restrict ourselves to the generator set $E_{L}$. 
In ref. [16], the generator set is chosen as the general enveloping algebra of HeisenbergWeyl Lie algebra. Let us first define the fundamental generator set $E^{0}$ to be the collection of all the creation and annihilation operators

$$
E^{0}:=\bigcup_{i}\left\{\hat{a}_{i}^{\dagger}, \hat{a}_{i}, \hat{\mathbb{I}}\right\}
$$

Here index $i$ stands for different creation and annihilation operators (in our context, $i$ may stand for $\vec{k}$ and the superscript $R$ and $L$.). $\hat{\mathbb{I}}$ satisfies $\left[\hat{a}_{i}, \hat{a}_{j}^{\dagger}\right]=\hat{\mathbb{I}} \delta_{i j}$ and $\hat{\mathbb{I}} \hat{e}=\hat{e}$ for $\forall \hat{e} \in E^{0}$. This fundamental operator set forms a Heisenberg-Weyl Lie algebra. Because this generator set is not big enough ref. [16] extends it to a larger set $E$ by

$$
E:=\bigcup_{n}\left(E^{0}\right)^{n}, \quad \text { with }\left(E^{0}\right)^{n}:=\left\{\hat{M}^{i_{1} i_{2} \cdots i_{n}}=: \hat{e}_{i_{1}} \hat{e}_{i_{2}} \cdots \hat{e}_{i_{n}}: \mid \forall \hat{e}_{i_{1}}, \hat{e}_{i_{2}}, \cdots, \hat{e}_{i_{n}} \in E^{0}\right\} .
$$

Here the ": :" stands for the normal ordering, e.g., : $\hat{a}_{i} \hat{a}_{j}^{\dagger}:=\hat{a}_{j}^{\dagger} \hat{a}_{i}$ for $\forall i, j$. In the definition (C.2), $\hat{e}_{i_{1}}, \hat{e}_{i_{2}}, \cdots, \hat{e}_{i_{n}}$ do not need to be different from each others. This extended operator set forms the general enveloping algebra of Heisenberg-Weyl Lie algebra. Any generator $\hat{T}(s)$ can be expand it by basis $E$ as follows:

$$
\hat{T}(s)=T_{0}(s) \hat{\mathbb{I}}+\sum_{i} Y_{i}(s) \hat{M}^{i}+\sum_{i j} Y_{i j}(s) \hat{M}^{i j}+\cdots+\sum_{i_{1} i_{2} \cdots i_{n}} Y_{i_{1} i_{2} \cdots i_{n}}(s) \hat{M}^{i_{1} i_{2} \cdots i_{n}}+\cdots .
$$

Here $T_{0}(s), Y_{i}(s), Y_{i j}(s), \cdots$ are complex numbers, $\hat{M}^{i}, \hat{M}^{i j}, \cdots$ are the elements in $E$ except for $\hat{\mathbb{I}}$. Then the Finsler structure expressed in the basis (C.2) is given by [16]

$$
\left.F\right|_{E}=\ell_{s}\left[\sum_{i}\left\|Y_{i}(t)\right\|+2 \sum_{i j}\left\|Y_{i j}(t)\right\|+\cdots+n \sum_{i_{1} i_{2} \cdots i_{n}}\left\|Y_{i_{1} i_{2} \cdots i_{n}}(t)\right\|+\cdots\right] .
$$

Here $\ell_{s}$ is a dimensionless positive constant. The meaning of $\|\cdot\|$ will clarified later on. For the continuous index case, the summation in the right-hand of eq. (C.4) should be replaced by integration. Here the index $E$ is added into $F$ to explicitly show that the right-hand of eq. (C.4) is valid only when we use the set $E$ to expand $\hat{T}(s)$.

In this paper, the generator set $E_{L}$ is neither $E$ nor its subsect, we cannot directly use formula (C.4) to obtain the functional form of the Finsler structure in the basis $E_{L}$ as the functional form of a Finsler structure depends on the basis. However, the generator set $E_{L}$ is just the linear combinations of some elements in $E$,

$$
\left[\begin{array}{c}
\hat{L}_{+}^{(\vec{k})} \\
\hat{L}_{-}^{(\vec{k})} \\
\hat{L}_{0}^{(\vec{k})}
\end{array}\right]=\left[\begin{array}{c}
1,0,0,0,0 \\
0,1,0,0,0 \\
0,0, \frac{1}{2}, \frac{1}{2}, \frac{1}{2}
\end{array}\right]\left[\begin{array}{c}
\hat{a}^{R \dagger}(\vec{k}) \hat{a}^{L \dagger}(\vec{k}) \\
\hat{a}^{R}(\vec{k}) \hat{a}^{L}(\vec{k}) \\
\hat{a}^{R \dagger}(\vec{k}) \hat{a}^{R}(\vec{k}) \\
\hat{a}^{L \dagger}(\vec{k}) \hat{a}^{L}(\vec{k}) \\
\hat{\mathbb{I}}
\end{array}\right]
$$

Let us consider a generator $\hat{T}(s)$ expanded it in the basis $E_{L}$

$$
\hat{T}(s)=\int \mathrm{d}^{d-1} k\left[\alpha_{+}(s, \vec{k}) \hat{L}_{+}^{(\vec{k})}+\alpha_{0}(s, \vec{k}) \hat{L}_{0}^{(\vec{k})}+\alpha_{-}(s, \vec{k}) \hat{L}_{-}^{(\vec{k})}\right] .
$$


Using the basis transformation formula in eq. (A.5) of ref. [16], we find that the functional form of the Finsler structure is

$$
\begin{aligned}
\left.F\right|_{E_{L}} & =\frac{2 \ell_{s} \Sigma_{d-1}}{(2 \pi)^{d-1}} \int \mathrm{d}^{d-1} k\left[\left\|\alpha_{+}(s, \vec{k})\right\|+\left\|\alpha_{-}(s, \vec{k})\right\|+\frac{1}{2}\left\|\alpha_{0}(s, \vec{k})\right\|+\frac{1}{2}\left\|\alpha_{0}(s, \vec{k})\right\|\right] \\
& =\ell_{0} \Sigma_{d-1} \int \mathrm{d}^{d-1} k\left[\left\|\alpha_{+}(s, \vec{k})\right\|+\left\|\alpha_{-}(s, \vec{k})\right\|+\left\|\alpha_{0}(s, \vec{k})\right\|\right]
\end{aligned}
$$

where we define that $\ell_{0}=2 \ell_{s} /(2 \pi)^{d-1}$.

The notation $\|\cdot\|$ was introduced in ref. [16]. Let's explain again why it is used in the Finsler structure (C.4). For a complex number $Y^{I}=\rho^{I} e^{i \theta^{I}}$ one may want to use $\left\|Y^{I}\right\|=\rho^{I}$ but this will lead to an inconsistent: the "rotation" caused by $\theta^{I}$ will change the operator $\hat{O}(s)$ but it does not change the complexity. One simple modification will be

$$
\left\|Y^{I}(s)\right\|=\rho^{I}\left(\left|\cos \theta^{I}\right|+\left|\sin \theta^{I}\right|\right)=\left|\operatorname{Re} Y^{I}(s)\right|+\left|\operatorname{Im} Y^{I}(s)\right| .
$$

Another possibility is [16]

$$
\left\|Y^{I}(s)\right\|:=\left|\operatorname{Re} Y^{I}(s)\right|+\left|\theta^{I}(s)\right| \cdot\left|\operatorname{Im} Y^{I}(s)\right| .
$$

where $\theta(0) \in[-\pi, \pi)$ and $\theta(s)$ is continuous for $s \in[0,1]$. In this paper, we choose the (C.9) as we can see that it can give the linear growth rate of the complexity at the late time limit.

\section{Complexity of operator generated by $\boldsymbol{E}_{L}$}

In this appendix, we will give the method to compute the complexity for any element in operators set $\mathcal{U}$ which is generated by generator set (4.23). Any operator in $\mathcal{U}$ can be parameterized uniquely by three complex-valued functions $\gamma_{+}(\vec{k}), \gamma_{-}(\vec{k})$ and $\gamma_{0}(\vec{k})$ by eq. (4.49). In order to find its complexity, we have to compute the lengths of all curves connecting $\hat{U}$ and identity in $\mathcal{U}$, and then find the minimal value of them. In $\mathcal{U}$, any curve starting from identity can be given by an $s$-dependent operator $\hat{O}(s)$ as

$$
\hat{O}(s)=\overleftarrow{P} \exp \left[\int_{0}^{s} \hat{T}(\tilde{s}) \mathrm{d} \tilde{s}\right]
$$

where

$$
\hat{T}(\tilde{s})=\int \mathrm{d}^{d-1} k\left[\alpha_{+}(\tilde{s}, \vec{k}) \hat{L}_{+}^{(\vec{k})}+\alpha_{0}(\tilde{s}, \vec{k}) \hat{L}_{0}^{(\vec{k})}+\alpha_{-}(\tilde{s}, \vec{k}) \hat{L}_{-}^{(\vec{k})}\right] .
$$

As $\left[\hat{T}\left(\tilde{s}_{1}\right), \hat{T}\left(\tilde{s}_{2}\right)\right] \neq 0$ in general when $s_{1} \neq s_{2}$, the time-order operator cannot be neglected. Different choices of functions $\left\{\alpha_{ \pm}(\tilde{s}, \vec{k}), \alpha_{0}(\tilde{s}, \vec{k})\right\}$ give different curves. We need this curve to end at $\hat{U}$ when $s=1$, i.e., $\hat{O}(1)=\hat{U}$. Let us find the relationship between $\left\{\gamma_{ \pm}(\vec{k}), \gamma_{0}(\vec{k})\right\}$ defined in eq. (4.49) and $\left\{\alpha_{ \pm}(s, \vec{k}), \alpha_{0}(s, \vec{k})\right\}$ when we require that $\hat{O}(1)=\hat{U}$.

It is more convenient to consider the problem in the discrete momentum system. As the elements with different momentum in (D.1) are commutative to each others, we can see that

$$
\hat{O}(s):=\prod_{\vec{k}_{i}} \hat{O}_{\vec{k}_{i}}(s)
$$


where

$$
\begin{aligned}
\hat{O}_{\vec{k}_{i}}(s) & :=\overleftarrow{P} \exp \left[\int_{0}^{s} \hat{T}_{\vec{k}_{i}}(\tilde{s}) \mathrm{d} \tilde{s}\right] \\
\hat{T}_{\vec{k}_{i}}(s) & =\alpha_{+}\left(\tilde{s}, \vec{k}_{i}\right) \hat{L}_{+}^{\left(\vec{k}_{i}\right)}+\alpha_{0}\left(\tilde{s}, \vec{k}_{i}\right) \hat{L}_{0}^{\left(\vec{k}_{i}\right)}+\alpha_{-}\left(\tilde{s}, \vec{k}_{i}\right) \hat{L}_{-}^{\left(\vec{k}_{i}\right)}
\end{aligned}
$$

The operator $\hat{O}_{\vec{k}_{i}}(s)$ has also a normal decomposition by three functions $b_{\vec{k}_{i}}(s), c_{\vec{k}_{i}}(s)$ and $d_{\vec{k}_{i}}(s)$

$$
\hat{O}_{\vec{k}_{i}}(s)=\exp \left[b_{\vec{k}_{i}}(s) \hat{L}_{+}^{\left(\vec{k}_{i}\right)}\right] \exp \left[c_{\vec{k}_{i}}(s) \hat{L}_{0}^{\left(\vec{k}_{i}\right)}\right] \exp \left[d_{\vec{k}_{i}}(s) \hat{L}_{-}^{\left(\vec{k}_{i}\right)}\right]
$$

Differentiating both (D.4) and (D.6) with respective to $s$, we have

$$
\begin{aligned}
\hat{T}_{\vec{k}_{i}} \hat{O}_{\vec{k}_{i}}= & b_{\vec{k}_{i}}^{\prime} \hat{L}_{+}^{\left(\vec{k}_{i}\right)} \exp \left[b_{\vec{k}_{i}} \hat{L}_{+}^{\left(\vec{k}_{i}\right)}\right] \exp \left[c_{\vec{k}_{i}} \hat{L}_{0}^{\left(\vec{k}_{i}\right)}\right] \exp \left[d_{\vec{k}_{i}} \hat{L}_{-}^{\left(\vec{k}_{i}\right)}\right] \\
& +c_{\vec{k}_{i}}^{\prime} \exp \left[b_{\vec{k}_{i}} \hat{L}_{+}^{\left(\vec{k}_{i}\right)}\right] \hat{L}_{0}^{\left(\vec{k}_{i}\right)} \exp \left[c_{\vec{k}_{i}} \hat{L}_{0}^{\left(\vec{k}_{i}\right)}\right] \exp \left[d_{\vec{k}_{i}} \hat{L}_{-}^{\left(\vec{k}_{i}\right)}\right] \\
& +d_{\vec{k}_{i}}^{\prime} \exp \left[b_{\vec{k}_{i}} \hat{L}_{+}^{\left(\vec{k}_{i}\right)}\right] \exp \left[c_{\vec{k}_{i}} \hat{L}_{0}^{\left(\vec{k}_{i}\right)}\right] \hat{L}_{-}^{\left(\vec{k}_{i}\right)} \exp \left[d_{\vec{k}_{i}} \hat{L}_{-}^{\left(\vec{k}_{i}\right)}\right] .
\end{aligned}
$$

There is a very useful formula when we compute the right hand of (D.7). For any two operators $\hat{A}, \hat{B}$, let us define $\left[{ }^{(0)} A, B\right]:=B$ and $\left[{ }^{(n+1)} A, B\right]:=\left[A,\left[{ }^{(n)} A, B\right]\right]$. Then we can find

$$
e^{\hat{A}} \hat{B} e^{-\hat{A}}=\sum_{n=0}^{\infty} \frac{1}{n !}\left[{ }^{(n)} \hat{A}, \hat{B}\right] .
$$

With this formula and the commutation relation (4.25), eq. (D.7) becomes

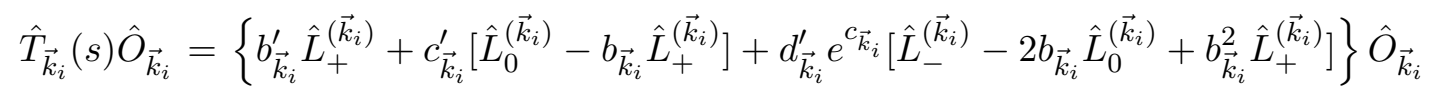

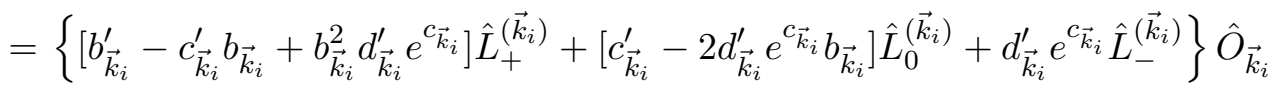

$$
\begin{aligned}
& =\left[\alpha_{+}\left(s, \vec{k}_{i}\right) \hat{L}_{+}^{\left(\vec{k}_{i}\right)}+\alpha_{0}\left(s, \vec{k}_{i}\right) \hat{L}_{0}^{\left(\vec{k}_{i}\right)}+\alpha_{-}\left(s, \vec{k}_{i}\right) \hat{L}_{-}^{\left(\vec{k}_{i}\right)}\right] \hat{O}_{\vec{k}_{i}} \text {. }
\end{aligned}
$$

Thus we obtain the following differential equations

$$
\begin{aligned}
b_{\vec{k}_{i}}^{\prime}(s) & =\alpha_{+}\left(s, \vec{k}_{i}\right)+\alpha_{0}\left(s, \vec{k}_{i}\right) b_{\vec{k}_{i}}(s)+b_{\vec{k}_{i}}^{2}(s) \alpha_{-}\left(s, \vec{k}_{i}\right), \\
c_{\vec{k}_{i}}^{\prime}(s) & =\alpha_{0}\left(s, \vec{k}_{i}\right)+2 b_{\vec{k}_{i}}(s) \alpha_{-}\left(s, \vec{k}_{i}\right), \\
d_{\vec{k}_{i}}^{\prime}(s) & =\alpha_{-}\left(s, \vec{k}_{i}\right) e^{-c_{\vec{k}_{i}}} .
\end{aligned}
$$

They should satisfy the following boundary conditions:

$$
\begin{aligned}
& b_{\vec{k}_{i}}(0)=c_{\vec{k}_{i}}(0)=d_{\vec{k}_{i}}(0)=0, \\
& b_{\vec{k}_{i}}(1)=\gamma_{+}\left(\vec{k}_{i}\right), \quad c_{\vec{k}_{i}}(1)=\ln \left(\gamma_{0}\left(\vec{k}_{i}\right)\right), \quad d_{\vec{k}_{i}}(1)=\gamma_{-}\left(\vec{k}_{i}\right) .
\end{aligned}
$$

The first line comes from the requirement $\hat{O}_{\vec{k}_{i}}(0)=I$ and the second line comes from $\hat{O}(1)=\hat{U}$ or $\hat{O}_{\vec{k}_{i}}(1)=\hat{U}_{\vec{k}_{i}}$, where $\hat{U}_{\vec{k}_{i}}$ is the discrete form of eq. (4.49):

$$
\hat{U}=\prod_{i} \hat{U}_{\vec{k}_{i}}
$$


with

$$
\hat{U}_{\vec{k}_{i}}:=\exp \left[\gamma_{+}\left(\vec{k}_{i}\right) \hat{L}_{+}^{\left(\vec{k}_{i}\right)}\right] \exp \left[\ln \gamma_{0}\left(\vec{k}_{i}\right) \hat{L}_{0}^{\left(\vec{k}_{i}\right)}\right] \exp \left[\gamma_{-}\left(\vec{k}_{i}\right) \hat{L}_{-}^{\left(\vec{k}_{i}\right)}\right] .
$$

Based on the function form in eq. (C.4), the complexity for a particular operator $\hat{U}$ defined in eq. (4.49) can be obtained by following optimization problem

$$
\begin{aligned}
& \Sigma_{d-1}^{-1} \mathcal{C}\left(\hat{U}\left[\gamma_{+}(\vec{k}), \gamma_{-}(\vec{k}), \gamma_{0}(\vec{k})\right]\right) \\
& \quad=\min \left\{\ell_{0} \int \mathrm{d}^{d-1} k \int_{0}^{1} \mathrm{~d} s\left[\left\|\alpha_{+}(s, \vec{k})\right\|+\left\|\alpha_{0}(s, \vec{k})\right\|+\left\|\alpha_{-}(s, \vec{k})\right\|\right]\right\}
\end{aligned}
$$

with the restrictions given by eq. (D.9) and eq. (D.10). As these restrictions are independent for different $\vec{k}$, we can further write eq. (D.13) as

$$
\begin{aligned}
& \Sigma_{d-1}^{-1} \mathcal{C}\left(\hat{U}\left[\gamma_{+}(\vec{k}), \gamma_{-}(\vec{k}), \gamma_{0}(\vec{k})\right]\right) \\
& \quad=\ell_{0} \int \mathrm{d}^{d-1} k\left[\min \int_{0}^{1} \mathrm{~d} s\left\{\left\|\alpha_{+}(s, \vec{k})\right\|+\left\|\alpha_{0}(s, \vec{k})\right\|+\left\|\alpha_{-}(s, \vec{k})\right\|\right\}\right]
\end{aligned}
$$

As the differential equations (D.9) is highly nonlinear, for general values of $\gamma_{+}(\vec{k}), \gamma_{-}(\vec{k})$ and $\gamma_{0}(\vec{k})$, the optimization problem (D.14) is not easy to solve. However, it is possible to find the complexity presented in eqs. (4.52) and (4.51). As the eq. (4.52) finds the minimal length in all the possible values of $\gamma_{-}(\vec{k})$ and $\gamma_{0}(\vec{k})$, the two of three functions $\alpha_{ \pm}(s, \vec{k})$ and $\alpha_{0}(s, \vec{k})$ will be free. We can choose that $\alpha_{-}(s, \vec{k})$ and $\alpha_{0}(s, \vec{k})$ are free. Then eqs. (D.14) becomes,

$$
\begin{aligned}
\Sigma_{d-1}^{-1} \mathcal{C}\left(\left|\operatorname{TFD}\left(t_{L}, t_{R}\right)\right\rangle,|0\rangle\right)= & \ell_{0} \int \mathrm{d}^{d-1} k\left[\operatorname { m i n } \int _ { 0 } ^ { 1 } \mathrm { d } s \left\{\left\|\alpha_{0}(s, \vec{k})\right\|+\left\|\alpha_{-}(s, \vec{k})\right\|\right.\right. \\
& \left.\left.+\left\|b_{\vec{k}}^{\prime}-\alpha_{0}(s, \vec{k}) b_{\vec{k}}+b_{\vec{k}}^{2} \alpha_{-}(s, \vec{k})\right\|\right\}\right],
\end{aligned}
$$

for arbitrary functions $\alpha_{-}^{(\vec{k})}, \alpha_{0}^{(\vec{k})}$ and $b_{\vec{k}_{i}}$ with $b_{\vec{k}_{i}}(0)=0$ and $b_{\vec{k}_{i}}(1)=\gamma_{+}(\vec{k})$. When $t=0$ in eq. (4.51), the solution of (D.15) can be obtain by the following method. As $\gamma_{+}(\vec{k}) \in \mathbb{R}$ and $0<\gamma_{+}(\vec{k}) \leq 1$, we can naturally expect that the solution of eq. (D.15) is given in the case $b_{\vec{k}}(s) \in \mathbb{R}$ and $\left|b_{\vec{k}}(s)\right| \leq 1$. Then one can see that

$$
\begin{aligned}
& \int_{0}^{1} \mathrm{~d} s\left\{\left\|\alpha_{0}^{(\vec{k})}(s)\right\|+\left\|\alpha_{-}^{(\vec{k})}(s)\right\|+\left\|b_{\vec{k}}^{\prime}(s)-\alpha_{0}(s, \vec{k}) b_{\vec{k}}(s)-b_{\vec{k}}^{2}(s) \alpha_{-}(s, \vec{k})\right\|\right\} \\
& \geq \int_{0}^{1} \mathrm{~d} s\left\{\left\|b_{\vec{k}}(s) \alpha_{0}^{(\vec{k})}(s)\right\|+\left\|b_{\vec{k}}^{2}(s) \alpha_{-}^{(\vec{k})}(s)\right\|+\left\|b_{\vec{k}}^{\prime}(s)-\alpha_{0}(s, \vec{k}) b_{\vec{k}}(s)-b_{\vec{k}}^{2}(s) \alpha_{-}(s, \vec{k})\right\|\right\} \\
& \geq \int_{0}^{1} \mathrm{~d} s\left\{\left\|b_{\vec{k}}(s) \alpha_{0}^{(\vec{k})}(s)+b_{\vec{k}}^{2}(s) \alpha_{-}^{(\vec{k})}(s)+b_{\vec{k}}^{\prime}(s)-\alpha_{0}(s, \vec{k}) b_{\vec{k}}(s)-b_{\vec{k}}^{2}(s) \alpha_{-}(s, \vec{k})\right\|\right\} \\
& =\int_{0}^{1} \mathrm{~d} s\left|b_{\vec{k}}^{\prime}(s)\right| \geq\left|\int_{0}^{1} b_{\vec{k}}^{\prime}(s) \mathrm{d} s\right|=\left|\gamma_{+}(\vec{k})\right| .
\end{aligned}
$$

The final equality can be satisfied only when $\alpha_{0}(s, \vec{k})=\alpha_{-}(s, \vec{k})=0$ and $b_{\vec{k}}^{\prime}(s)<0$ or $b_{\vec{k}}^{\prime}(s)>0$ for $\forall s \in(0,1)$. When $t_{L}+t_{R} \neq 0$, we see $\operatorname{Im} \gamma_{+}(\vec{k}) \neq 0$. In this case, we have to separate every variable into the real part and the imaginary part firstly. Then we use 
the definition in eq. (C.9) to convert $\|\cdot\|$ into the usual absolute symbol. After that, we can use the Euler-Lagrange equation to find the minimal value in eq. (D.15). The result still shows that the minimal value can be reached if $\alpha_{0}(s, \vec{k})=\alpha_{-}(s, \vec{k})=0$. Hence, we find that,

$$
\Sigma_{d-1}^{-1} \mathcal{C}\left(\left|\operatorname{TFD}\left(t_{L}, t_{R}\right)\right\rangle,|0\rangle\right)=\ell_{0} \int \mathrm{d}^{d-1} k\left\|\gamma_{+}(\vec{k})\right\|
$$

Open Access. This article is distributed under the terms of the Creative Commons Attribution License (CC-BY 4.0), which permits any use, distribution and reproduction in any medium, provided the original author(s) and source are credited.

\section{References}

[1] M. Van Raamsdonk, Building up spacetime with quantum entanglement, Gen. Rel. Grav. 42 (2010) 2323 [arXiv: 1005. 3035] [INSPIRE].

[2] S. Ryu and T. Takayanagi, Holographic derivation of entanglement entropy from AdS/CFT, Phys. Rev. Lett. 96 (2006) 181602 [hep-th/0603001] [INSPIRE].

[3] J. Maldacena and L. Susskind, Cool horizons for entangled black holes, Fortsch. Phys. 61 (2013) 781 [arXiv: 1306.0533] [INSPIRE].

[4] T. Faulkner, M. Guica, T. Hartman, R.C. Myers and M. Van Raamsdonk, Gravitation from Entanglement in Holographic CFTs, JHEP 03 (2014) 051 [arXiv:1312.7856] [INSPIRE].

[5] A. Einstein and N. Rosen, The particle problem in the general theory of relativity, Phys. Rev. 48 (1935) 73 [INSPIRE].

[6] L. Susskind, Computational Complexity and Black Hole Horizons, Fortsch. Phys. 64 (2016) 44 [arXiv: 1403.5695] [INSPIRE].

[7] L. Susskind, Entanglement is not enough, Fortsch. Phys. 64 (2016) 49 [arXiv:1411.0690] [INSPIRE].

[8] J.M. Maldacena, Eternal black holes in anti-de Sitter, JHEP 04 (2003) 021 [hep-th/0106112] [INSPIRE].

[9] A.R. Brown, D.A. Roberts, L. Susskind, B. Swingle and Y. Zhao, Complexity, action and black holes, Phys. Rev. D 93 (2016) 086006 [arXiv: 1512.04993] [InSPIRE].

[10] M. Alishahiha, Holographic Complexity, Phys. Rev. D 92 (2015) 126009 [arXiv:1509.06614] [INSPIRE].

[11] O. Ben-Ami and D. Carmi, On volumes of subregions in holography and complexity, JHEP 11 (2016) 129 [arXiv:1609.02514] [INSPIRE].

[12] J. Couch, W. Fischler and P.H. Nguyen, Noether charge, black hole volume and complexity, JHEP 03 (2017) 119 [arXiv: 1610.02038] [INSPIRE].

[13] D. Stanford and L. Susskind, Complexity and Shock Wave Geometries, Phys. Rev. D 90 (2014) 126007 [arXiv:1406 . 2678] [INSPIRE].

[14] A.R. Brown, D.A. Roberts, L. Susskind, B. Swingle and Y. Zhao, Holographic Complexity Equals Bulk Action?, Phys. Rev. Lett. 116 (2016) 191301 [arXiv:1509.07876] [INSPIRE].

[15] S. Chapman, M.P. Heller, H. Marrochio and F. Pastawski, Towards Complexity for Quantum Field Theory States, arXiv: 1707.08582 [INSPIRE]. 
[16] R.-Q. Yang, A Complexity for Quantum Field Theory States and Application in Thermofield Double States, arXiv:1709.00921 [INSPIRE].

[17] P. Caputa, N. Kundu, M. Miyaji, T. Takayanagi and K. Watanabe, Anti-de Sitter Space from Optimization of Path Integrals in Conformal Field Theories, Phys. Rev. Lett. 119 (2017) 071602 [arXiv: 1703.00456] [INSPIRE].

[18] P. Caputa, N. Kundu, M. Miyaji, T. Takayanagi and K. Watanabe, Liouville action as path-integral complexity: from continuous tensor networks to AdS/CFT, JHEP 11 (2017) 097 [arXiv: 1706. 07056] [inSPIRE].

[19] K. Shizume, T. Nakajima, R. Nakayama and Y. Takahashi, Quantum computational Riemannian and sub-Riemannian geodesics, Prog. Theor. Phys. 127 (2012) 997 [InSPIRE].

[20] M.A. Nielsen, A geometric approach to quantum circuit lower bounds, Quantum Info. Comput. 6 (2006) 213.

[21] M.R. Dowling and M. A. Nielsen, The geometry of quantum computation, Quantum Info. Comput. 8 (2008) 861.

[22] R.A. Jefferson and R.C. Myers, Circuit complexity in quantum field theory, JHEP 10 (2017) 107 [arXiv: 1707.08570] [INSPIRE].

[23] D. Carmi, S. Chapman, H. Marrochio, R.C. Myers and S. Sugishita, On the time dependence of holographic complexity, JHEP 11 (2017) 188 [arXiv:1709.10184] [INSPIRE].

[24] S. Lloyd, Ultimate physical limits to computation, Nature 406 (2000) 1047.

[25] R.-G. Cai, S.-M. Ruan, S.-J. Wang, R.-Q. Yang and R.-H. Peng, Action growth for AdS black holes, JHEP 09 (2016) 161 [arXiv:1606.08307] [INSPIRE].

[26] R.-Q. Yang, Strong energy condition and complexity growth bound in holography, Phys. Rev. D 95 (2017) 086017 [arXiv: 1610.05090] [INSPIRE].

[27] R.-G. Cai, M. Sasaki and S.-J. Wang, Action growth of charged black holes with a single horizon, Phys. Rev. D 95 (2017) 124002 [arXiv:1702.06766] [INSPIRE].

[28] D. Carmi, R.C. Myers and P. Rath, Comments on holographic complexity, JHEP 03 (2017) 118 [arXiv: 1612.00433] [INSPIRE].

[29] S. Chapman, H. Marrochio and R.C. Myers, Complexity of formation in holography, JHEP 01 (2017) 062 [arXiv: 1610.08063] [INSPIRE].

[30] R.-Q. Yang, C. Niu and K.-Y. Kim, Surface counterterms and regularized holographic complexity, JHEP 09 (2017) 042 [arXiv:1701.03706] [inSPIRE].

[31] K. Parattu, S. Chakraborty, B.R. Majhi and T. Padmanabhan, A Boundary Term for the Gravitational Action with Null Boundaries, Gen. Rel. Grav. 48 (2016) 94 [arXiv: 1501.01053] [INSPIRE].

[32] L. Lehner, R.C. Myers, E. Poisson and R.D. Sorkin, Gravitational action with null boundaries, Phys. Rev. D 94 (2016) 084046 [arXiv: 1609.00207] [InSPIRE].

[33] F. Hopfmüller and L. Freidel, Gravity Degrees of Freedom on a Null Surface, Phys. Rev. D 95 (2017) 104006 [arXiv: 1611.03096] [INSPIRE].

[34] I. Jubb, J. Samuel, R. Sorkin and S. Surya, Boundary and Corner Terms in the Action for General Relativity, Class. Quant. Grav. 34 (2017) 065006 [arXiv: 1612.00149] [InSPIRE]. 
[35] A. Reynolds and S.F. Ross, Divergences in Holographic Complexity, Class. Quant. Grav. 34 (2017) 105004 [arXiv: 1612.05439] [INSPIRE].

[36] G. Hayward, Gravitational action for spacetimes with nonsmooth boundaries, Phys. Rev. D 47 (1993) 3275.

[37] T. Hartman and J. Maldacena, Time evolution of entanglement entropy from black hole interiors, JHEP 05 (2013) 014 [arXiv: 1303.1080] [INSPIRE].

[38] M. Miyaji, T. Numasawa, N. Shiba, T. Takayanagi and K. Watanabe, Distance between Quantum States and Gauge-Gravity Duality, Phys. Rev. Lett. 115 (2015) 261602 [arXiv: 1507.07555] [INSPIRE].

[39] M. Sinamuli and R.B. Mann, Geons and the Quantum Information Metric, Phys. Rev. D 96 (2017) 026014 [arXiv: 1612.06880] [INSPIRE].

[40] I. Bengtsson, Geometry of quantum states: an introduction to quantum entanglement, Cambridge University Press, New York U.S.A. (2006).

[41] A. Klimov, A group-theoretical approach to quantum optics: models of atom-field interactions, Wiley-VCH, Weinheim Germany (2009).

[42] A.R. Brown and L. Susskind, The Second Law of Quantum Complexity, arXiv:1701.01107 [INSPIRE].

[43] K. Hashimoto, N. Iizuka and S. Sugishita, Time evolution of complexity in Abelian gauge theories, Phys. Rev. D 96 (2017) 126001 [arXiv:1707.03840] [InSPIRE].

[44] M.M. Qaemmaqami, Complexity growth in minimal massive 3D gravity, Phys. Rev. D 97 (2018) 026006 [arXiv: 1709.05894] [INSPIRE].

[45] L.C.B. Crispino, A. Higuchi and G.E.A. Matsas, The Unruh effect and its applications, Rev. Mod. Phys. 80 (2008) 787 [arXiv:0710.5373] [INSPIRE]. 